\title{
A method for computing unsteady fully nonlinear interfacial waves
}

\author{
by \\ Helmer André Friis, John Grue, Enok Palm and Per Olav Rusås \\ Mechanics Division, Department of mathematics, \\ University of Oslo, Norway
}

\begin{abstract}
We derive a time stepping method for unsteady fully nonlinear two-dimensional motion of a two-layer fluid. Essential parts of the method are: use of Taylor series expansions of the prognostic equations, application of spacial finite difference formulae of high order, and application of Cauchy's theorem to solve the Laplace equation, where the latter is found to be advantageous in avoiding instability. The method is computationally very efficient. The model is applied to investigate unsteady transcritical two-layer flow over a bottom topography. We are able to simulate a set of laboratory experiments described by Melville and Helfrich (1987) on this problem, finding a very good agreement between the fully nonlinear model and the experiments, where bad agreement with weakly nonlinear KdV theories was reported. The unsteady transcritical regime is identified. In this regime, we find that an upstream undular bore is generated when the speed of the geometry is less than a certain value, which somewhat exceeds the critical speed. In the remaining regime, a train of solitary waves is generated upstream. In both cases a corresponding elevation of constant level behind the geometry is developed. We also perform a detailed investigation of upstream generation of solitary waves by a moving geometry, finding that wave trains with amplitude comparable to the thickness of the thinner layer are generated. The results indicate that weakly nonlinear theories in many cases have quite limited applications in modelling unsteady transcritical two-layer flows, and that a fully nonlinear method in general is required.
\end{abstract}

\section{Introduction}

In 1896, Fridtjov Nansen and his crew returned from their three years long journey to the Arctic Ocean with a wealth of scientific observations and descriptions of unexplained natural phenomena; one of them the dead water problem. Nansen discussed his observations on this phenomenon with Vilhelm Bjerknes, who attributed the wave resistance to internal waves generated by the ship, at the interface separating a layer of fresh water and heavier salt water. Today, one hundred years after the FRAM expedition, knowledge of flows due to internal waves, their origin and propagation, is important for many reasons, in addition to the dead water problem. Some examples are flows in fjords and at sills, breaking of internal waves and mixing processes in the ocean, motion in coastal water and sub-surface waves in a layered ocean. An important aspect of the latter relates to oil exploration in deep water, with operation performed from ships or oil platforms floating at the sea surface, connected to subsea drilling or production via long cables. Knowledge of currents in the ocean, which may be induced by internal waves, may be of importance for the design of such concepts, in addition to the wave effects at the ocean surface. Dynamics of internal waves is also important in dimensioning of subwater bridges, which 
have been proposed across Norwegian fjords. Further description of the scientific results of the FRAM expedition may be found in Eliassen (1982).

This study is in particular motivated by needs relating to the two latter problems. We want to investigate propagation properties of internal waves, their generation and interaction with a bottom topography or a geometry in the ocean; our formulation is therefore unsteady. Observations in nature, see e.g. Farmer and Smith (1980), Apel et al. (1985), and laboratory experiments, see e.g. Koop and Butler (1981), Melville and Helfrich (1987), show that internal or interfacial waves may have quite large amplitudes due to the relatively small differences in density, such that the flow in many cases is outside the range of validity of weakly nonlinear theories. Motivated by these and similar observations our formulation is fully nonlinear.

To simplify the problem we consider two-dimensional flow of a two-layer fluid. This enables us to derive a time stepping procedure using a pseudo-Lagrangian method, where at each time step the position of the interphase and the quantity $\phi_{1}-\mu \phi_{2}$ are found, where $\phi_{1}$ and $\phi_{2}$ denote the velocity potentials in the lower and upper layer, respectively, and $\mu=\rho_{2} / \rho_{1}$, where $\rho$ denotes the corresponding fluid density. The discrete version of the prognostic equations are obtained by Taylor series expansions including several terms, as advocated by Dold and Peregrine (1985), Dold (1992), who studied nonlinear free surface flows. This leads to very efficient computations.

The Eulerian velocity fields in the layers are obtained by solving the Laplace equation at each time step. It turns out that accurate solution of the Laplace equation is crucial to an algoritm for computing interfacial flows. Earlier works on time evolution of nonlinear interfacial waves have applied singularity distributions along the interface to solve the Laplace equation, see e.g. Baker, Meiron and Orzag (1982), Roberts (1983), Eliassen and Fjørtoft (1992). During the development of the present method we have investigated the performance of vortex or dipole distributions, but encountered problems due to numerical instability of the solution even for moderate nonlinearity of the waves. This was also the conclusion of the algoritm described by Baker et al. (1982), and, at the onset of the algoritm described by Roberts (1983). Roberts was, however, able to make modifications of the scheme removing the instabilities in his investigations. We have found it advantageous to seek a different method than using singularity distributions directly to solve the Laplace equation, and have chosen to employ Cauchy's integral theorem for this purpose. This method has been applied in several works on evolution of nonlinear free surface waves, see e.g. Vinje and Brevig (1981) and Dold and Peregrine (1985), and is reported to give less problems with regard to numerical instability than other algorithms developed for flows with free boundaries. We find that this is true also for computations of interfacial waves, which partly is explained by a stability analysis ( 33.2$)$, where we find that our scheme is neutrally stable for linear flows.

While Baker et al. (1982) basically used their method to simulate free surface flows, Roberts (1983) simulated steadily progressing interfacial waves, and Eliassen and Fjørtoft (1992) computed interfacial waves and roll up of vortices developing from an initial disturbance. Their method was also formulated for flows in three dimensions.

Here, we apply the model to investigate transcritical two-layer flow over a bottom topography. This issue has been studied by means of hydraulic nonlinear theory or weakly nonlinear dispersive models (Baines 1984, Grimshaw and Smyth 1986, Miles 1979,1981, Melville and Helfrich 1987, Zhu, Wu and Yates 1987), assuming that the amplitude is small, which, however, may be a severe restriction in many cases. We are able to simulate a set of laboratory experiments described by Melville and Helfrich (1987) on transcritical 
flow, which to our knowledge not yet have been confirmed by theoretical and numerical models. In this set of experiments, where the elevation of the interface is rather large, we find a striking agreement between the fully nonlinear model and the experiments, where severe disagreement with weakly nonlinear KdV theories was reported. Furthermore, we investigate upstream generation of solitary waves when the depth ratio between the two fluids is one to four. In all cases here we find that trains of solitary waves with amplitudes comparable to the thickness of the thinner layer are generated, irrespective of the nonlinearity of the forcing (the volume under the geometry cannot be too small). This means that weakly nonlinear theories are inadequate in the examples considered. Upstream influence in a two-layer fluid has its counterpart in free surface flows, see e.g. $\mathrm{Wu}$ (1987) for a review.

The literature on nonlinear internal waves is rather extensive. The first works date back to Keulegan (1953) and Long (1956) who investigated interfacial solitary wave solutions exploiting weakly nonlinear Boussinesq equations and the Korteweg-de Vries equation, finding sech ${ }^{2}$-profiles. Benjamin (1967) investigated weakly nonlinear internal waves when one of the layers is infinitely deep, allowing also for a continous stratification, finding solitary waves of algebraic form. He also investigated flows due to two infinite layers of different but constant densities separated by an intermediate layer where the density can vary. The latter problem was at the same time considered by Davis and Acrivos (1967). Later on, Ono (1975) generalized the work of Benjamin to include also unsteady behaviour of algebraic solitary waves, deriving an equation which later was termed the Benjamin-Ono (BO) equation. To bridge the gap between the shallow water and the BO equations Joseph (1977) and Kubota, Ko and Dobbs (1978) derived the finite-depth theory.

During the past couple of decades there are published several works on strongly nonlinear periodic progressing waves, which for sufficiently large amplitude may be overhanging (Holyer 1979, Vanden-Broeck 1980, Meiron and Saffman 1983). There are also several works on strongly nonlinear interfacial solitary waves, which, like periodic waves may be overhanging for large amplitude, see e.g. Pullin and Grimshaw (1988). Furthermore, solitary waves are found to have broadening effect due to a limited depth of the thicker layer (Amick and Turner 1986). We have used our method to recompute weakly nonlinear interfacial waves, steep periodic wave trains and nonlinear solitary waves, arriving at the results described in the cited works.

It is relevant to mention the rather broad activity on internal waves, see e.g. Staquet and Sommeria (1996).

In $\S 2$ the mathematical formulation is given, including description of the time stepping procedure and the solution procedure of the Laplace equation. (We note that $\S 2.2$ describing the time stepping procedure, in many steps necessarily is quite similar to the descriptions by Roberts (1983) and Eliassen and Fjørtoft (1992).) §3 describes the numerical implementation, stability, accuracy and convergence of the method, and how we prevent breakdown of the simulations, which can be caused by the physical KelvinHelmoltz instability in flows with a finite velocity jump at the interface. In $\S 4$ we compare fully nonlinear solitary wave solutions with available experiments and weakly nonlinear theories. $\S 5$ is devoted to transcritical two-layer flow over a bottom topography, upstream influence and generation of solitary waves. Finally, $\S 6$ is a conclusion. 


\section{Mathematical formulation}

\subsection{The boundary value problem}

We consider two-dimensional motion of two fluid layers of infinite horizontal extension under the action of gravity, with the gravitation force along the negative vertical direction. The lower fluid layer has thickness $h_{1}$ at rest and constant density $\rho_{1}$, and the upper layer has thickness $h_{2}$ at rest and constant density $\rho_{2}$, where $\rho_{2}$ is smaller than $\rho_{1}$. Hereafter, index 1 refers to the lower fluid, and index 2 to the upper. A coordinate system $O-x y$ is introduced with the $x$-axis along the interface at rest and the $y$-axis pointing upwards. Unit vectors $\mathbf{i}, \mathbf{j}$ are introduced accordingly. We assume that the two fluids are homogenous and incompressible and that the motion in each of the layers is irrotational such that the velocities may be obtained by potential theory, i.e.

$$
\mathbf{v}_{1}=u_{1} \mathbf{i}+v_{1} \mathbf{j}=\nabla \phi_{1}, \quad \mathbf{v}_{2}=u_{2} \mathbf{i}+v_{2} \mathbf{j}=\nabla \phi_{2},
$$

where $\phi_{1}$ and $\phi_{2}$ satisfy the Laplace equation in their respective domains.

The kinematic boundary condition at the interface $I$ separating the fluids is given by

$$
\frac{\partial \phi_{1}}{\partial n}=\frac{\partial \phi_{2}}{\partial n} \quad \text { at } \quad I
$$

where $\partial / \partial n$ means differentiation along the unit normal $\mathbf{n}$ of the interface. The dynamic boundary condition at the interface is obtained by balancing the pressure difference with the interfacial tension, giving

$$
p_{1}-p_{2}=\frac{\sigma}{R_{I}} \quad \text { at } \quad I
$$

Here $\sigma$ denotes the coefficient of interfacial tension, $R_{I}$ the radius of curvature of the interface, and the pressure in each of the layers is given by the Bernoulli equation, i.e.

$$
\frac{p_{j}}{\rho_{j}}+\frac{\partial \phi_{j}}{\partial t}+\frac{1}{2} \mathbf{v}_{j}^{2}+g y=0, \quad j=1,2
$$

We shall in the model account for a restrained or moving body in one of the fluids. Here we develop the equations for a geometry in the lower layer, but note that the equations may easily be modified to also account for a geometry in the upper layer. For a geometry moving with translatory velocity $\mathbf{V}$, the kinematic condition at its boundary $B$ gives

$$
\frac{\partial \phi_{1}}{\partial n}=\mathbf{V} \cdot \mathbf{n} \quad \text { at } \quad B
$$

If $\mathbf{V}=0, \partial \phi_{1} / \partial n=0$ at $B$. The lower boundary of layer 1 , outside a possible geometry, is horizontal, and the kinematic boundary condition there gives

$$
\frac{\partial \phi_{1}}{\partial y}=0 \quad \text { at } \quad y=-h_{1}
$$

As boundary condition on top of the upper fluid, which in realistic problems is a free surface, we may in the cases under investigation justify the simplifying rigid lid approximation, i.e.

$$
\frac{\partial \phi_{2}}{\partial y}=0 \quad \text { at } \quad y=h_{2}
$$

To close the boundary value problem the motion is either assumed to be periodic in the $x$-direction or disappear for $x \rightarrow \pm \infty$.

A schematic view of the problem is shown in figure 1. 


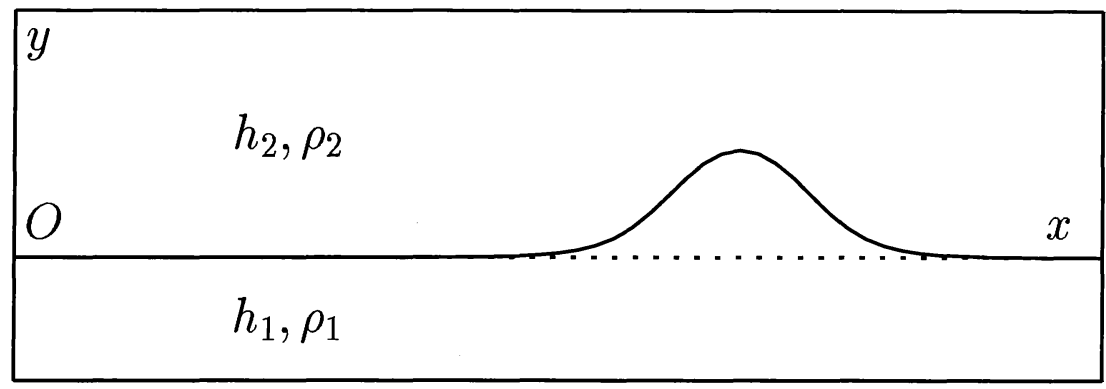

Figure 1: Two-dimensional interfacial flow.

\section{$2.2 \quad$ Time stepping procedure}

A time stepping procedure for the interface and the flow in the fluids may be derived from the kinematic and dynamic boundary conditions at the interface. We adopt a Lagrangian method where pseudo Lagrangian particles are introduced on the interface, each with a weighted velocity $\mathbf{v}_{\times}$defined by

$$
\mathbf{v}_{\times}=(1-\alpha) \mathbf{v}_{1}+\alpha \mathbf{v}_{2}
$$

where $0 \leq \alpha \leq 1$. A pseudo particle coincides with a particle at the interface in the lower fluid if $\alpha=0$, and with a particle at the interface in the upper fluid if $\alpha=1$. We have applied different values of $\alpha$ in testing the method and find that best performance is achieved when $\alpha$ is chosen such that a typical horizontal velocity of the pseudo particles is as small as possible. A pseudo Lagrangian derivative is next introduced by

$$
\frac{D_{\times}}{d t}=\frac{\partial}{\partial t}+\mathbf{v}_{\times} \cdot \nabla
$$

To determine the position $\mathbf{R}=(X, Y)$ of a pseudo particle we have the following relation

$$
\frac{D_{\times} \mathbf{R}}{d t}=\mathbf{v}_{\times}
$$

We next consider the dynamic boundary condition at the interface. The pressure in the fluids may by means of (9) be written

$$
\frac{p_{j}}{\rho_{j}}+\frac{D_{\times} \phi_{j}}{d t}-\mathbf{v}_{\times} \cdot \mathbf{v}_{j}+\frac{1}{2} \mathbf{v}_{j}^{2}+g y=0, \quad j=1,2
$$

Introducing (11) into the dynamic boundary condition (3) we find

$$
\frac{D_{\times}\left(\phi_{1}-\mu \phi_{2}\right)}{d t}=\mathbf{v}_{\times} \cdot\left(\mathbf{v}_{1}-\mu \mathbf{v}_{2}\right)-\frac{1}{2}\left(\mathbf{v}_{1}^{2}-\mu \mathbf{v}_{2}^{2}\right)-(1-\mu) g Y-\frac{\sigma}{\rho_{1} R_{I}} \text { at } I
$$

where $\mu=\rho_{2} / \rho_{1}$. This motivates for introducing at the interface $I$

$$
\psi=\phi_{1}-\mu \phi_{2}
$$

For later use (see §2.3) we also introduce,

$$
\Upsilon=\phi_{1}+\phi_{2}
$$


and derive equations for $\psi, \Upsilon_{\xi}$ and $\phi_{1 \nu}=\phi_{2 \nu}$ at the interface, and thereafter find $\phi_{1 \xi}$ and $\phi_{2 \xi}$ from

$$
\begin{gathered}
\phi_{1 \xi}=\frac{1}{1+\mu}\left(\psi_{\xi}+\mu \Upsilon_{\xi}\right) \\
\phi_{2 \xi}=\frac{1}{\mu}\left(\phi_{1 \xi}-\psi_{\xi}\right)
\end{gathered}
$$

The equations (10) and (12) contain sufficient information to integrate $\mathbf{R}$ and $\psi$ forward in time. It is, however, an advantage to apply also higher order derivatives of (10) and (12) in a time stepping procedure for $\mathbf{R}$ and $\psi$. Following Dold and Peregrine (1985), who studied free surface waves, the prognostic equations for $\mathbf{R}$ and $\psi$ are obtained by Taylor series expansions, as follows

$$
\begin{gathered}
\mathbf{R}(t+\Delta t)=\mathbf{R}(t)+\mathbf{v}_{\times}(t) \Delta t+\frac{1}{2 !} \frac{D_{\times} \mathbf{v}_{\times}}{d t}(t)(\Delta t)^{2}+\frac{1}{3 !} \frac{D_{\times}^{2} \mathbf{v}_{\times}}{d t^{2}}(t)(\Delta t)^{3}+\ldots \\
\psi(t+\Delta t)=\psi(t)+\frac{D_{\times} \psi}{d t}(t) \Delta t+\frac{1}{2 !} \frac{D_{\times}^{2} \psi}{d t^{2}}(t)(\Delta t)^{2}+\frac{1}{3 !} \frac{D_{\times}^{3} \psi}{d t^{3}}(t)(\Delta t)^{3}+\ldots
\end{gathered}
$$

This procedure has an error of the order $(\Delta t)^{n+1} /(n+1)$ ! at each time step when terms up to the $n$th derivative are included in the expansions, which is an essential improvement compared to an algorithm based on first-derivatives only. In the computations we truncate the series after $n=7$. Procedures of how to evaluate $D_{\times}^{m} \mathbf{v}_{\times} / d t^{m}(m=1, \ldots, 6)$ and $D_{\times}^{m} \psi / d t^{m}(m=1, \ldots, 7)$ are described at the end of $\S 2.3$ and in appendix A.

\subsection{Solution of the Laplace equation}

As noted in the Introduction, accurate solution of the Laplace equation is crucial to an algoritm for computing interfacial flows, and we here apply Cauchy's integral theorem for this purpose. Invoking complex analysis we introduce the complex variable $z=x+i y$ and complex velocities $q_{j}(z)=u_{j}-i v_{j}, j=1,2$. Since $q_{j}$ are analytic functions of $z$, we have by use of Cauchy's integral theorem for $z^{\prime}$ outside the respective fluid layers

$$
\oint_{C_{1}} \frac{q_{1} d z}{z-z^{\prime}}=0, \quad \oint_{C_{2}} \frac{q_{2} d z}{z-z^{\prime}}=0
$$

The contours $C_{j}(j=1,2)$ are composed of the interface, the rigid horizontal boundaries of the fluid layers, and $C_{1}$ contains the contour of the bottom topography. In addition, $C_{j}$ contains vertical boundaries, either in accordance with the periodicity of the flow, or at infinity. The integrals over these parts of $C_{j}$ vanish due to the conditions described in $\S 2.1$ after eq. (7). The integration is clockwise along $C_{1}$ and counterclockwise along $C_{2}$.

The rigid wall conditions (6) and (7) at $y=-h_{1}, h_{2}$ are accounted for by applying the method of images. For a point $z$ at the interface, the images about the rigid walls at $y=-h_{1}$ and $y=h_{2}$ are given by, respectively,

$$
\tilde{z}_{1}=z^{*}-2 i h_{1}, \quad \tilde{z}_{2}=z^{*}+2 i h_{2}
$$

where a star denotes complex conjungate. Analytical continuation of the complex velocities outside the rigid boundaries of the respective fluid domains are obtained by

$$
q_{j}\left(\tilde{z}_{j}\right)=\left[q_{j}(z)\right]^{*}
$$


(19) then gives

$$
\begin{aligned}
& \int_{I} \frac{q_{2}(z) d z}{z-z^{\prime}}-\int_{I} \frac{q_{2}(z)^{*} d z^{*}}{z^{*}+2 i h_{2}-z^{\prime}}=0 \\
& \int_{I+B} \frac{q_{1}(z) d z}{z-z^{\prime}}-\int_{I+B} \frac{q_{1}(z)^{*} d z^{*}}{z^{*}-2 i h_{1}-z^{\prime}}=0
\end{aligned}
$$

Letting $z^{\prime}$ approach $I$ and $B$ from outside each of the fluids and applying the Plemelj formula we obtain from (22)-(23):

$$
\begin{array}{rlr}
-\pi i q_{2}\left(z^{\prime}\right) & =P V \int_{I} \frac{q_{2}(z) d z}{z^{\prime}-z}+\int_{I} \frac{q_{2}(z)^{*} d z^{*}}{z^{*}+2 i h_{2}-z^{\prime}} \quad(z \text { on } I) \\
\pi i q_{1}\left(z^{\prime}\right) & =P V \int_{I} \frac{q_{1}(z) d z}{z^{\prime}-z}+\int_{I} \frac{q_{1}(z)^{*} d z^{*}}{z^{*}-2 i h_{1}-z^{\prime}} & \\
& +\int_{B} \frac{q_{1}(z) d z}{z^{\prime}-z}+\int_{B} \frac{q_{1}(z)^{*} d z^{*}}{z^{*}-2 i h_{1}-z^{\prime}} \quad(z \text { on } I) \\
\pi i q_{1}\left(z^{\prime}\right)= & \int_{I} \frac{q_{1}(z) d z}{z^{\prime}-z}+\int_{I} \frac{q_{1}(z)^{*} d z^{*}}{z^{*}-2 i h_{1}-z^{\prime}} \\
+ & P V \int_{B} \frac{q_{1}(z) d z}{z^{\prime}-z}+\int_{B} \frac{q_{1}(z)^{*} d z^{*}}{z^{*}-2 i h_{1}-z^{\prime}} \quad(z \text { on } B)
\end{array}
$$

where $P V$ denotes principal value. Only the real part of the principal value integrals in (24)-(26) are singular.

Following the method by Dold and Peregrine (1985) for free surface flows, we introduce a $\xi$-variable as coordinate along the interface, and tangential and normal derivatives of the potentials at $I$ and $B$ by

$$
q_{j}=\left(\phi_{j s}-i \phi_{j n}\right) z_{s}^{*}=\left(\phi_{j \xi}-i \phi_{j \nu}\right) \frac{z_{\xi}^{*}}{\left|z_{\xi}\right|^{2}}, \quad j=1,2
$$

where ()$_{s}=\partial / \partial s$ denotes differentiation along the arclength $s$ of $I,()_{n}=\partial / \partial n$, ()$_{\xi}=\partial / \partial \xi,()_{\nu}=\partial / \partial \nu$. We hereafter work with the scaled tangential and normal velocities $\phi_{j \xi}=\phi_{j s}\left|z_{\xi}\right|$ and $\phi_{j \nu}=\phi_{j n}\left|z_{\xi}\right|$.

We then subtract (24) from (25), multiply by $z_{\xi}^{\prime}$ and apply (27). From the imaginary part of the result we obtain the following equation for $\Upsilon_{\xi}=\phi_{1 \xi}+\phi_{2 \xi}$

$$
\begin{aligned}
\pi \Upsilon_{\xi}\left(\xi^{\prime}\right) & =\frac{2}{\mu+1} \int_{I} \operatorname{Im}\left(\frac{z_{\xi}^{\prime}}{z^{\prime}-z}\right) \psi_{\xi} d \xi+\frac{\mu-1}{\mu+1} \int_{I} \operatorname{Im}\left(\frac{z_{\xi}^{\prime}}{z^{\prime}-z}\right) \Upsilon_{\xi} d \xi \\
& +\int_{I} \operatorname{Re}\left(\frac{z_{\xi}^{\prime}}{z^{*}-2 i h_{1}-z^{\prime}}-\frac{z_{\xi}^{\prime}}{z^{*}+2 i h_{2}-z^{\prime}}\right) \phi_{1 \nu} d \xi \\
& +\frac{1}{1+\mu} \int_{I} \operatorname{Im}\left(\frac{z_{\xi}^{\prime}}{z^{*}-2 i h_{1}-z^{\prime}}+\frac{z_{\xi}^{\prime}}{z^{*}+2 i h_{2}-z^{\prime}}\right) \psi_{\xi} d \xi \\
& +\frac{1}{1+\mu} \int_{I} \operatorname{Im}\left(\mu \frac{z_{\xi}^{\prime}}{z^{*}-2 i h_{1}-z^{\prime}}-\frac{z_{\xi}^{\prime}}{z^{*}+2 i h_{2}-z^{\prime}}\right) \Upsilon_{\xi} d \xi \\
& +\int_{B} \operatorname{Re}\left(-\frac{z_{\xi}^{\prime}}{z^{\prime}-z}+\frac{z_{\xi}^{\prime}}{z^{*}-2 i h_{1}-z^{\prime}}\right) \phi_{1 \nu} d \xi \\
& +\int_{B} \operatorname{Im}\left(\frac{z_{\xi}^{\prime}}{z^{\prime}-z}+\frac{z_{\xi}^{\prime}}{z^{*}-2 i h_{1}-z^{\prime}}\right) \phi_{1 \xi} d \xi
\end{aligned}
$$


for $z^{\prime}$ on $I$. To obtain (28) we have also used the kinematic condition (2) at the interface. We note that (28) does not contain principal value integrals.

Next, multiplying (24) and (25) by $z_{\xi}^{\prime}$ and taking the real part, we may combine the resulting equations to give

$$
\begin{aligned}
\pi(1+\mu) \phi_{1 \nu}\left(\xi^{\prime}\right) & =P V \int_{I} \operatorname{Re}\left(\frac{z_{\xi}^{\prime}}{z^{\prime}-z}\right) \psi_{\xi} d \xi+(1-\mu) \int_{I} \operatorname{Im}\left(\frac{z_{\xi}^{\prime}}{z^{\prime}-z}\right) \phi_{1 \nu} d \xi \\
& +\frac{1}{1+\mu} \int_{I} \operatorname{Re}\left(\frac{z_{\xi}^{\prime}}{z^{*}-2 i h_{1}-z^{\prime}}+\mu \frac{z_{\xi}^{\prime}}{z^{*}+2 i h_{2}-z^{\prime}}\right) \psi_{\xi} d \xi \\
& +\frac{\mu}{1+\mu} \int_{I} \operatorname{Re}\left(\frac{z_{\xi}^{\prime}}{z^{*}-2 i h_{1}-z^{\prime}}-\frac{z_{\xi}^{\prime}}{z^{*}+2 i h_{2}-z^{\prime}}\right) \Upsilon_{\xi} d \xi \\
& -\int_{I} \operatorname{Im}\left(\frac{z_{\xi}^{\prime}}{z^{*}-2 i h_{1}-z^{\prime}}-\mu \frac{z_{\xi}^{\prime}}{z^{*}+2 i h_{2}-z^{\prime}}\right) \phi_{1 \nu} d \xi \\
& +\int_{B} \operatorname{Re}\left(\frac{z_{\xi}^{\prime}}{z^{\prime}-z}+\frac{z_{\xi}^{\prime}}{z^{*}-2 i h_{1}-z^{\prime}}\right) \phi_{1 \xi} d \xi \\
& +\int_{B} \operatorname{Im}\left(\frac{z_{\xi}^{\prime}}{z^{\prime}-z}-\frac{z_{\xi}^{\prime}}{z^{*}-2 i h_{1}-z^{\prime}}\right) \phi_{1 \nu} d \xi
\end{aligned}
$$

for $z^{\prime}$ on $I$, where (2) is applied.

Finally, multiplying (26) by $z_{\xi}^{\prime}$ and taking the imaginary part, we obtain

$$
\begin{aligned}
\pi \phi_{1 \xi}\left(\xi^{\prime}\right) & =\int_{I} R e\left(-\frac{z_{\xi}^{\prime}}{z^{\prime}-z}+\frac{z_{\xi}^{\prime}}{z^{*}-2 i h_{1}-z^{\prime}}\right) \phi_{1 \nu} d \xi \\
& +\frac{1}{1+\mu} \int_{I} \operatorname{Im}\left(\frac{z_{\xi}^{\prime}}{z^{\prime}-z}+\frac{z_{\xi}^{\prime}}{z^{*}-2 i h_{1}-z^{\prime}}\right) \psi_{\xi} d \xi \\
& +\frac{\mu}{1+\mu} \int_{I} \operatorname{Im}\left(\frac{z_{\xi}^{\prime}}{z^{\prime}-z}+\frac{z_{\xi}^{\prime}}{z^{*}-2 i h_{1}-z^{\prime}}\right) \Upsilon_{\xi} d \xi \\
& +P V \int_{B} \operatorname{Re}\left(-\frac{z_{\xi}^{\prime}}{z^{\prime}-z}+\frac{z_{\xi}^{\prime}}{z^{*}-2 i h_{1}-z^{\prime}}\right) \phi_{1 \nu} d \xi \\
& +\int_{B} \operatorname{Im}\left(\frac{z_{\xi}^{\prime}}{z^{\prime}-z}+\frac{z_{\xi}^{\prime}}{z^{*}-2 i h_{1}-z^{\prime}}\right) \phi_{1 \xi} d \xi
\end{aligned}
$$

for $z^{\prime}$ on $B$.

The equations (28)-(30) determine $\Upsilon_{\xi}$ and $\phi_{1 \nu}$ at the interface $I$ and $\phi_{1 \xi}$ at the bottom topography $B$, since $\psi$ on $I$ and $\phi_{1 \nu}$ on $B$ are given. The velocities on both sides of the interface are finally found from (15), (16), (27).

The bottom topography in the lower fluid may be replaced by a submerged body. In this case the solution of (29)-(30) contains an arbitary circulation about the closed body surface, and the requirement of no circulation about the body contour, i.e.

$$
\int_{B} \phi_{1 \xi}(\xi) d \xi=0
$$

must be included as an additional equation.

We are now able to evaluate the terms containing the first derivatives in (17)-(18). In order to obtain the second and third order pseudo Lagrangian derivatives of $\mathbf{R}$ and $\psi$, we need to compute $\partial q_{j} / \partial t$ and $\partial^{2} q_{j} / \partial t^{2}$, which, by exploiting their analyticity, may 
be found from Cauchy's theorem, giving the same integral equations (24)-(26) as for the velocities $q_{j}$. Similar integral equations for $\partial \phi_{1 \nu} / \partial t, \partial \Upsilon_{\xi} / \partial t$ and $\partial^{2} \phi_{1 \nu} / \partial t^{2}, \partial^{2} \Upsilon_{\xi} / \partial t^{2}$ may be constructed by exploiting the relevant boundary conditions at the interface; the details are given in appendix A. Pseudo derivatives of the order four to seven are obtained by backwards differencing in time, based on the third order pseudo derivatives.

\subsection{The Boussinesq approximation}

When computing interfacial waves in e.g. the ocean, where $\mu$ is close to 1 , we may apply the Boussinesq approximation. We then keep $1-\mu=\epsilon$ in the bouyancy term of (12), but put $\mu=1$ in all other terms. For the particular choice $\alpha=1 / 2$ in eq. (8) we then obtain

$$
\frac{D_{\times} \mathbf{R}}{d t}=\mathbf{v}_{\times}, \quad \frac{D_{\times} \psi}{d t}=-\epsilon g Y+\frac{\sigma}{R_{I}} \quad \text { at } I
$$

The equations for determining $\Upsilon_{\xi}$ and $\phi_{1 \nu}$ also become simplified. In particular, for $h_{1}=h_{2}=\infty$ and no body geometry present in the fluid, we obtain

$$
\Upsilon_{\xi}^{\prime}=\frac{1}{\pi} \int_{I} \operatorname{Im}\left(\frac{z_{\xi}^{\prime}}{z^{\prime}-z}\right) \psi_{\xi} d \xi, \quad \phi_{1 \nu}^{\prime}=\frac{1}{2 \pi} P V \int_{I} R e\left(\frac{z_{\xi}^{\prime}}{z^{\prime}-z}\right) \psi_{\xi} d \xi
$$

which are valid for a nonlinear interface, and means that $\Upsilon_{\xi}$ and $\phi_{1 \nu}$ are determined by integrals of (the given) $\psi_{\xi}$. This was also noted by Eliassen and Fjørtoft (1992).

This result is in contrast to modelling nonlinear free surface waves $(\mu=0)$ in infinite water depth, where a set of equations must be solved to determine $\phi_{1 \nu}$ at each time step.

When $h_{1}$ and $h_{2}$ are finite, the Boussinesq approximation is still a gain, since fewer iterations are required in the solution procedure than when $\mu \neq 1$, see $\S 3.1$.

\section{Numerical aspects of the method}

\subsection{Implementation}

The functions in the problem are assumed to be smooth along the boundaries and at any point within the fluids. We discretize the interface by $N$ pseudo Lagrangian points and the body by $N_{B}$ points, where $\xi$ takes integer values at each point, i.e. $\xi=1,2, \ldots, N$ at $I$ and $\xi=1,2, \ldots, N_{B}$ at $B$. The integrals are approximated by the trapezoidal rule. This integration rule gives a spectrally accurate numerical integration, i.e. the error is exponentially small, when the integrand is periodic and has sufficiently smooth derivatives, see e.g. Shelley (1992). We find that the trapezoidal rule is well suited for the problems considered here. Following Dold and Peregrine (1985), the principal value integrals are evaluated by first expanding the integrand in a series in the vincity of the pole at $z=z^{\prime}$, and then carrying out the integration. For a smooth real function $f$ we have

$$
P V \int_{0}^{N} \frac{z_{\xi}^{\prime}}{z^{\prime}-z(\xi)} f(\xi) d \xi=\sum_{\xi=1}^{N}\left[\mathcal{A}\left(\xi^{\prime}, \xi\right)+i \mathcal{B}\left(\xi^{\prime}, \xi\right)\right] f(\xi)-f_{\xi}\left(\xi^{\prime}\right)
$$

where

$$
\mathcal{A}+i \mathcal{B}= \begin{cases}z_{\xi}^{\prime} /\left(z^{\prime}-z\right) & z \neq z^{\prime} \\ z_{\xi \xi} / 2 z_{\xi} & z=z^{\prime}\end{cases}
$$


The accuracy of the integration in (34) is determined by the numerical differentiation formula being applied. In most of the computations we apply a 10-points finite difference formula based on a 10th-order polynomial expansion with an error of the order $|\Delta \mathbf{R}|^{10}$. In the case of periodic motion we also in some examples obtain the derivatives from truncated Fourier series, giving the derivatives and the approximation to the integral in (34) with spectral accuracy. The discrete forms of the integral equations (28), (29) and (30) are given in appendix B.

To solve the equations we first invert (30) by Gaussian elimination, expressing $\phi_{1 \xi}$ at $B$ by the unknowns $\phi_{1 \nu}$ and $\Upsilon_{\xi}$ at $I$. (28)-(29) are then solved by iteration using the generalized conjugate residuals method, which is both robust and efficient. The organization of the equations for the unknowns at the interface on the form (28)-(29) is advantageous with respect to an efficient solution procedure. In order to minimize the round-off-error, all computations are performed by double precision arithmetics.

As described in $\S 2.2$ we use the expansions (17)-(18) to step $\mathbf{R}$ and $\psi$ forward in time, where the derivatives up to the third order are obtained from a combination between analytical formulae and solution of the integral equations. Further derivatives are found by use of 4 orders of backwards differencing. During the first 5 time steps of the computation the order of the backward differencing polynomial is gradually increased from 0 to 4 . The time steps are small in the beginning of the computations, and are gradually increased up to a constant value $\Delta t$.

In all numerical examples presented we neglect the interfacial tension, i.e $\sigma=0$ in the computations.

A regridding algoritm is applied to the pseudo particles in order to maintain an even distance between the node points at the interface during the calculations.

Mostly due to the Kelvin-Helmholtz instability (see §3.4) smoothing is required in order to maintain a stable solution. We use two smoothing formulae which both are based on a 15-points interpolation polynomial, one smooth 2-points periodic (sawtooth) disturbances from the grid, the other removes both 3-points and 2-points periodic disturbances. Both smoothing formulae remove very little energy from the waves.

\subsection{Stability of the linearized scheme. Dispersion relation.}

It is of interest to investigate the stability and the dispersion relation of the linearized scheme. We consider periodic perturbations $Y, \phi_{1}, \phi_{2}$, satisfying the linear versions of $(2),(10)$ and $(12)$ (with $\sigma=0)$ at $y=0$, i.e.

$$
\frac{\partial Y}{\partial t}=\frac{\partial \phi_{1}}{\partial y}, \quad \frac{\partial \psi}{\partial t}+(1-\mu) g Y=0
$$

There is no geometry in the fluids and we set $h_{1}=h_{2}=\infty$. From the integral equation (29) we find

$$
\pi(1+\mu) \frac{\partial \phi_{1}}{\partial y}=-P V \int_{-\infty}^{\infty} \frac{\psi_{x}}{x-x^{\prime}} d x
$$

where the principal value integral is the double sided Hilbert transform of $\psi_{x}$. By combining (37) with (36) we find

$$
\frac{\partial^{2} \psi}{\partial t^{2}}=\frac{g(1-\mu)}{\pi(1+\mu)} P V \int_{-\infty}^{\infty} \frac{\psi_{x}}{x-x^{\prime}} d x
$$


We consider a periodic interface and discretize the interface by $N$ points per wave length, giving for the discrete version of (38)

$$
\frac{\partial^{2} \psi\left(\xi^{\prime}\right)}{\partial t^{2}}=-\frac{g(1-\mu)}{\pi(1+\mu)}\left(\mathcal{A D}_{1}-\mathcal{D}_{2}\right) \psi, \quad \xi^{\prime}=1,2, \ldots, N
$$

where $\mathcal{A}$ is given by the periodic version of (35) and $\mathcal{D}_{1}, \mathcal{D}_{2}$ denote the operators for the first and second derivatives. To investigate the stability of the numerical scheme we seek solutions of $(39)$ on the form $\psi(x, t)=\hat{\psi}(x) e^{i \omega t}$ (and $Y(x, t)=\hat{Y}(x) e^{i \omega t}$ ) where $i \omega=\omega_{r}+i \omega_{i}$. This leads to the following eigenvalue problem

$$
\omega^{2} \hat{\psi}=\frac{g(1-\mu)}{\pi(1+\mu)}\left(\mathcal{A D}_{1}-\mathcal{D}_{2}\right) \hat{\psi}
$$

The solution of this problem, obtained by a standard method, contains a double set of $N$ non-zero eigensolutions corresponding to linear waves with wavenumbers $k=1,2, \ldots, N / 2$ and (eigen) frequencies $\omega=\omega_{i 1}, \omega_{i 2}, \ldots, \omega_{i N / 2}$, which may propagate in both horizontal directions. The first set has phase shift 0 , the other phase shift $\pi / 2$. We find that $\omega_{r}=0$ for all wavenumbers, both when the derivatives are obtained by the 10-points finite difference formula and by the truncated Fourier series, which means that the numerical scheme is neutrally stable for linear waves.

Next we consider the analytic and numerical dispersion relations of the problem, which are obtained by inserting $\psi=\operatorname{Re}\left(\hat{\psi} e^{i k x-i \omega t}\right)$ into (38) and (39), respectively ( $\omega$ denotes the frequency and $k$ the wave number). Noting that the double sided Hilbert transform of $e^{i k x}$ is determined by

$$
P V \int_{-\infty}^{\infty} \frac{e^{i k x}}{x-x^{\prime}} d x=i \pi e^{i k x^{\prime}}
$$

we find from (38) that the analytical dispersion relation becomes

$$
\omega^{2}=g k(1-\mu) /(1+\mu)
$$

When the periodic interface is discretized by $N$ points per wave length we obtain from (39) a numerical dispersion relation for wavenumbers up to $k=N / 2$, the Nyquist wave. This dispersion relation is obtained by differencing in two ways; either by the 10-points finite difference formula or by the truncated Fourier series. Since the tapezoidal rule yields a spectrally accurate approximation to the integral in (41) (see §3.1) we expect that the latter method gives a numerical dispersion relation with spectral accuracy, as also found by the computations. We note that the various dispersion relations are independent of the value of $\mu$, except for the prefactor on the right of (40), which is due to that we for the moment have set $h_{1}=h_{2}=\infty$. In fact, we recover the results for free surface waves $(\mu=0)$ which is discussed by Dold (1992).

In figure 2 we show the graph of $\omega^{2}$ versus $k$ for the analytical dispersion relation (42) and the two different numerical dispersion relations. (The numerical dispersion relation corresponding to the spectral derivatives coincides with the analytical dispersion relation for $k \leq N / 2$.) The numerical dispersion relation due to the 10 -points finite difference formula coincides with the analytical dispersion relation up to $k \sim N / 4$, but deviates somewhat for larger wavenumbers. This deviation is of minor importance, however, since we in the numerical simulations apply smoothing to remove short disturbances. From numerical experiments it turns out that the 10-points finite difference formula is adequate for the examples considered here. 


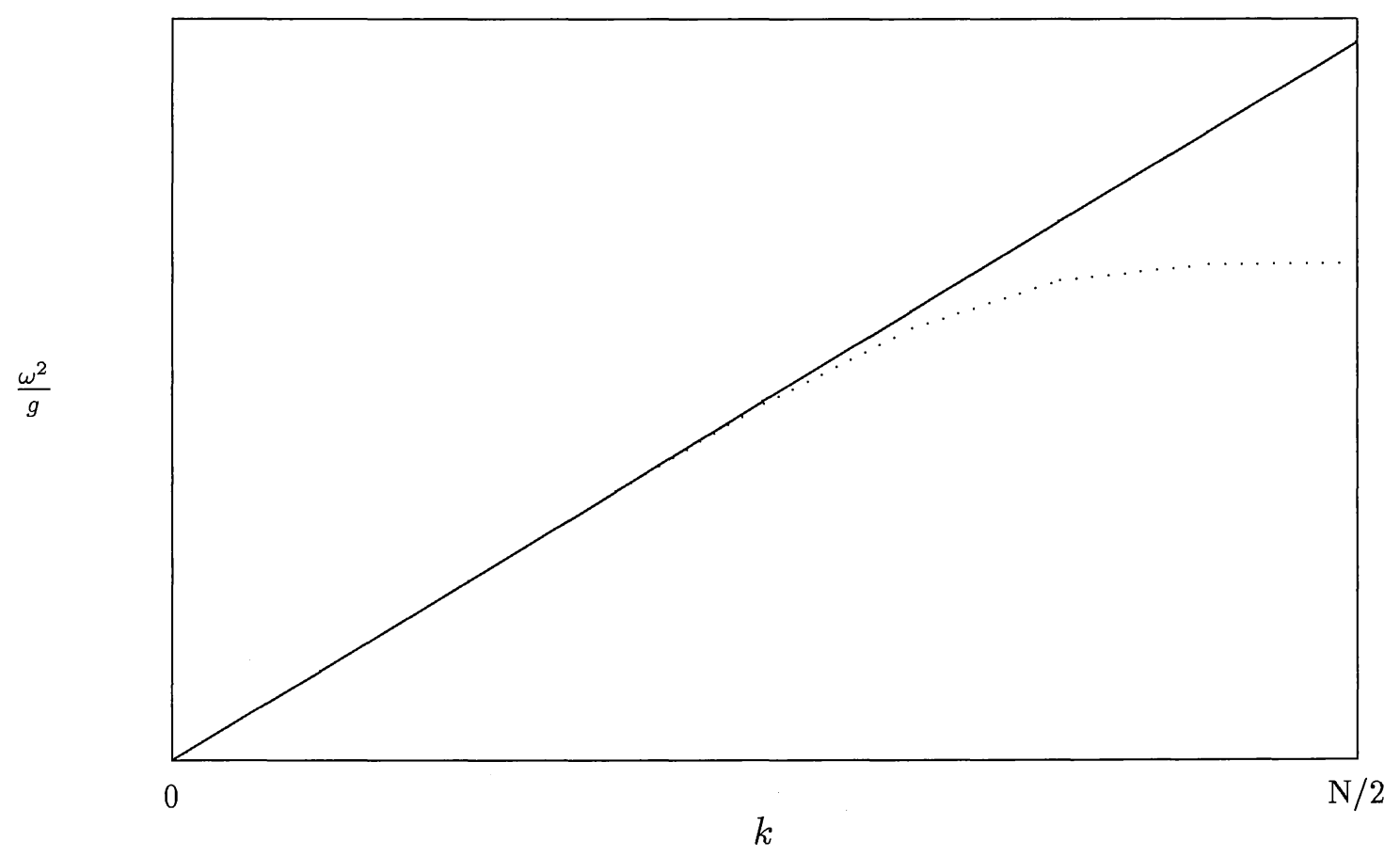

Figure 2: Numerical dispersion relation. Solid line: Analytical (42) and spectral method. Dotted line: 10-points finite difference formula used for numerical differentiation $(\mu=$ 0.1 .

\subsection{The effect of the Kelvin-Helmholtz instability}

When we apply the model to linear interfacial waves, we encounter no problems with regard to instability. Once the wave steepness becomes finite, however, the physical Kelvin-Helmholtz (K-H) instability due to the wave-induced velocity jump occurring at the interface is included in the model. This means that the numerical solution becomes unstable to disturbances with wavelength shorter than a treshold value, $\lambda_{K H}$, say, which prevents us for refining the space discretization below this value, if no smoothing is applied. The nonlinear computations show that $\lambda_{K H}$ has a marked increase with growing velocity jump at the interface. $\lambda_{K H}$ is also growing with increasing $\mu=\rho_{2} / \rho_{1}$. This increase is, however, moderate when $\mu$ is larger than about 0.2 , since the velocity shear due to the interfacial waves is decreasing with increasing $\mu$.

The $\mathrm{K}-\mathrm{H}$ instability due to interfacial waves in a real two-layer fluid, or a fluid with a localized vertical density variation, is in most cases limited by local nonlinearity, viscous effects and/or interfacial tension, which are effects that prevents breakdown of the large scale wave motion. Thus, the present model, where the effect of the K-H instability is suppressed, may provide a reasonable approach to large scale behaviour of interfacial waves, as long as $\lambda_{K H}$ is much smaller than the main length scales of the wave motion.

\subsection{Accuracy and convergence}

To measure the accuracy and the convergence of the method we investigate how quantities like wave form, volume of the wave, mean level of the interface and energy are conserved in some examples for waves propagating steadily along the interface.

The kinetic energy due to the motion in the fluid layers may, by applying the divergence 
theorem and the kinematic boundary conditions, be expressed as an integral along the interface. By adding the potential energy we find that the energy of the fluid motion is given by

$$
E=\frac{1}{2} \rho_{1} \int_{0}^{N} \psi(\xi) \phi_{1 \nu}(\xi) d \xi+\frac{1}{2} g \rho_{1}(1-\mu) \int_{0}^{N} Y^{2}(\xi) X_{\xi}(\xi) d \xi
$$

The initial profile and propagating velocity $c$ of the wave(s) are determined by applying the equations of $\S \S 2.2-2.3$ in a frame of reference moving with the speed of the wave, i.e. where $\partial / \partial t=-c \partial / \partial x$. The resulting nonlinear equations are solved by means of Newton's method.

In the first example we compute periodic nonlinear interfacial waves propagating during a time interval corresponding to four wave periods. The wave data are: $a k=0.345$ ( $a$ the wave amplitude, $k$ the wave number), $\mu=0.1$ and $h_{1}=h_{2}=\infty$. We show in tables 1 and 2 the relative error in the mean interfacial level $(\bar{Y})$ and the energy $(E)$, for various discretizations per wavelength $(N)$, time step $(\Delta t)$ and smoothing interval $(\Delta T)$. The tables clearly demonstrate convergence of the method with respect to increasing $N$ and decreasing $\Delta t$, that the smoothing interval $\Delta T$ cannot be too large, and that highest accuracy is obtained for relatively small $\Delta T$. The smallest relative error in these computations is as small as $3 \cdot 10^{-8}$. The relative errors in the wave form and the propagation speed are of the same order as for $\bar{Y}$ and $E$.

In the next example a solitary wave is considered, with $|Y|_{\max } / h_{2}=0.7, h_{1} / h_{2}=4$, $\mu=0.9$. The interface is discretized from the wave crest and out to horizontal positions $\pm 59.5 h_{2}$, where $\left|Y / h_{2}\right|$ becomes smaller than $10^{-10}$. The resulting wave is shown in figure 4b. Table 3 displays the relative error in volume and energy after the wave has propagated a distance $100 h_{2}$. Again the computations document convergence of the method, and that a relatively small smoothing interval $\Delta T$ gives a high accuracy. We note though that $\Delta t / \Delta T$ always should be a small number in the computations, in order to smooth as little as possible. The smallest relative error in these computations is $5 \cdot 10^{-6}$.

\section{Solitary waves. Comparison with experiments and weakly nonlinear theories}

One way of illustrating the usefulness of our fully nonlinear method is to compare solitary wave solutions with available laboratory measurements and results by asymptotic theories for such waves, i.e. $\mathrm{KdV}, \mathrm{BO}$ and finite-depth theories. In a work by Koop and Butler (1981) they described laboratory experiments on interfacial solitary waves using water above freon and compared their results with these asymptotic theories. They had, however, no fully nonlinear method available. In figure 3 we have reproduced the data set from their figure 10, displaying the half mean elevation of solitary waves, defined by $L=\int_{-\infty}^{\infty} Y d x /(2 a)$, as function of the maximal elevation $a=|Y|_{\text {max }}$, for $\mu=0.633$ and $h_{2} / h_{1}=5.086$. Due to the moderate value of $h_{2} / h_{1}$ we do not expect the BO equation to give good results in this comparison. The figure clearly shows that the finite-depth and the $\mathrm{KdV}$ theories have quite limited ranges of validity. In this example the finite-depth theory is good up to $a / h_{1}$ about 0.1 , while the $\mathrm{KdV}$ theory is relevant for somewhat larger $a / h_{1}$, up to about 0.2 . Koop and Butler also provided an extension of the KdV theory, which increased the domain of validity for the theory. However, for nondimensional amplitude of the order unity, which is a relevant elevation in many applications (see also 


\begin{tabular}{|c|c|c|c|}
\hline$N$ & $\omega \Delta T$ & $\bar{Y} / a$ & $\Delta E / E$ \\
\hline 20 & 2.0 & 0.00260000 & 0.00600000 \\
\hline 20 & 1.5 & 0.00000860 & 0.00000530 \\
\hline 20 & 1.0 & 0.00000160 & 0.00000170 \\
\hline 20 & 0.5 & 0.00000180 & 0.00000370 \\
\hline
\end{tabular}

\begin{tabular}{|c|c|c|c|}
\hline 30 & 0.5 & 0.00000030 & 0.00000015 \\
\hline 30 & 0.25 & 0.00000009 & 0.00000014 \\
\hline 30 & 0.1 & 0.00000014 & 0.00000025 \\
\hline 30 & 0.05 & 0.00000020 & 0.00000037 \\
\hline
\end{tabular}

\begin{tabular}{|c|c|c|c|}
\hline 60 & 0.1 & 0.00014000 & 0.00013000 \\
\hline 60 & 0.05 & 0.00000130 & 0.00000120 \\
\hline 60 & 0.01 & 0.00000002 & 0.00000003 \\
\hline
\end{tabular}

Table 1: Propagation of periodic interfacial waves (wave period $2 \pi / \omega$ ). Relative error after four wave periods in the mean interfacial level and energy $E$ vs. discretization $N$ and smooting interval $\omega \Delta T$. Time step $\omega \Delta t=0.01$. $a k=0.345, \mu=0.1, h_{1}=h_{2}=\infty$. 2-point smoothing for $N=20,30$. 3-point smoothing for $N=60$.

\begin{tabular}{|c|c|c|}
\hline$\omega \Delta t$ & $\bar{Y} / a$ & $\Delta E / E$ \\
\hline 0.5 & 0.0002100 & 0.0004000 \\
\hline 0.2 & 0.0000027 & 0.0000074 \\
\hline 0.1 & 0.0000012 & 0.0000024 \\
\hline 0.01 & 0.0000016 & 0.0000017 \\
\hline
\end{tabular}

Table 2: Same as table 1, but $\omega \Delta T=1.0, N=20,2$-point smoothing.

\begin{tabular}{|c|c|c|c|c|}
\hline$N$ & $\Delta t\left(g / h_{2}\right)^{1 / 2}$ & $\Delta T\left(g / h_{2}\right)^{1 / 2}$ & $\Delta V / V$ & $\Delta E / E$ \\
\hline 61 & 0.1 & 5.0 & 0.000300 & 0.001000 \\
\hline 81 & 0.1 & 3.0 & 0.000030 & 0.000090 \\
\hline 125 & 0.1 & 1.0 & 0.000005 & 0.000005 \\
\hline
\end{tabular}

\begin{tabular}{|l|l|l|l|l|}
\hline 81 & 3.0 & 3.0 & 0.002000 & 0.000900 \\
\hline 81 & 1.0 & 3.0 & 0.000200 & 0.000010 \\
\hline 81 & 0.5 & 3.0 & 0.000030 & 0.000090 \\
\hline
\end{tabular}

Table 3: Relative error in volume $V$ and energy $E$ due to solitary wave which has propagated a distance of $100 h_{2} .|Y|_{\max } / h_{2}=0.7, h_{1} / h_{2}=4, \mu=0.9$. 2-point smoothing. The wave profile is shown in figure $4 \mathrm{~b}$. 


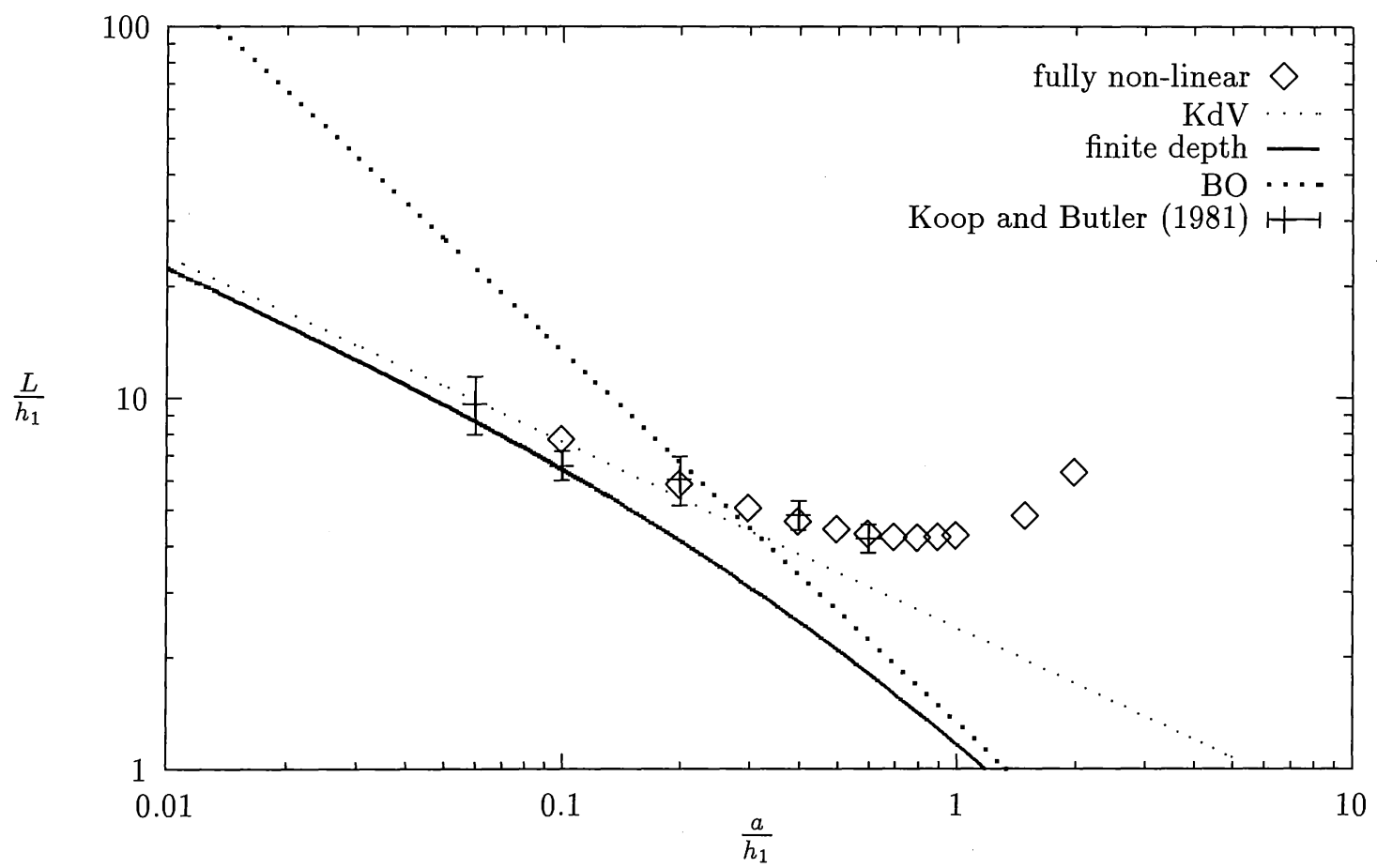

Figure 3: Solitary waves. Comparison between fully nonlinear theory $(\diamond)$, laboratory measurements by Koop and Butler (1981, figure 10) (vertical bars), KdV-theory (weak dotted line), BO-theory (fat dotted line), finite-depth theory (solid line). Vertical coordinate: Half mean elevation of solitary wave. Horizontal coordinate: Amplitude $a=|Y|_{\text {max }}$. $\mu=0.633, h_{2} / h_{1}=5.086$.

$\S 5)$, the results clearly demonstrate the shortcomings of the finite-depth and the $\mathrm{KdV}$ theories.

In figure 3 are also displayed results obtained by the fully nonlinear method, which show excellent comparison with the experimental measurements for the whole range of $a / h_{1}$. For small amplitudes our method agrees with the asymptotic theories. In the BO limit (results not shown here) discretization of the interface out to a very large $X$ coordinate is required. We note that the nondimensional area under the waves is increasing with the amplitude for $a / h_{1}>1$, which is due to the broadening effect occurring for a limited ratio between $h_{2}$ and $h_{1}$, see Amick and Turner (1986).

The fundamental differences between the fully nonlinear and the weakly nonlinear methods are further illustrated by the solitary wave profiles shown in figure 4, which are relevant for the applications described in the next section. We observe that the finitedepth, KdV and fully nonlinear methods are in approximate agreement for $|Y|_{\max } / h_{2}$ up to about 0.15 (figure 4a). For nondimensional amplitude of the order unity, however, the differences between the methods become remarkably large.

Figure 4 again illustrates that the $\operatorname{sech}^{2}$-solitons from the $\mathrm{KdV}$ theory are closer to the fully nonlinear solution than those due to the finite-depth theory, in spite of the relatively large ratio between $h_{2}$ and $h_{1}$ in these examples, which might lead us to believe that the opposite should be true. This somewhat surprising result has been explained by Segur and Hammack (1982) who found that the range of validity of the finite-depth theory with regard to nonlinearity is far more limited than that for the $\mathrm{KdV}$ theory, giving that the former theory produce unrealistic results much earlier than the latter. 


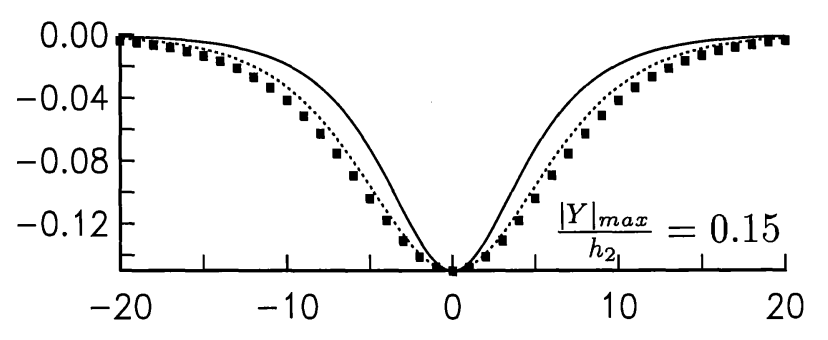

a)

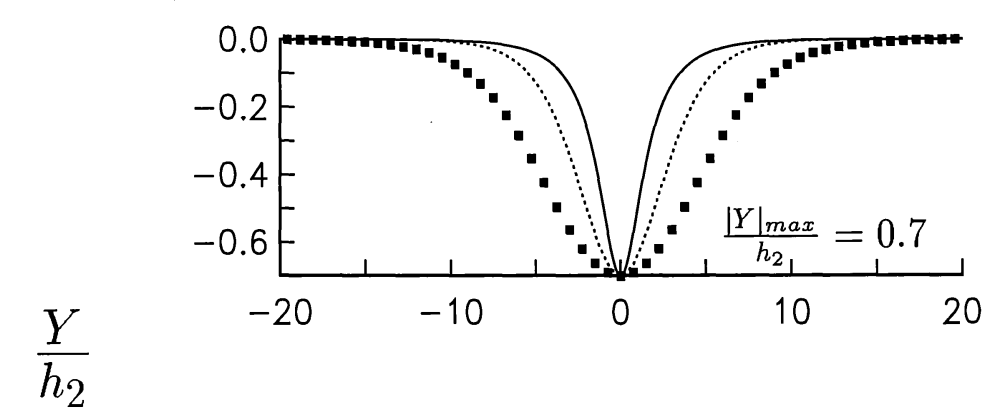

b)

)

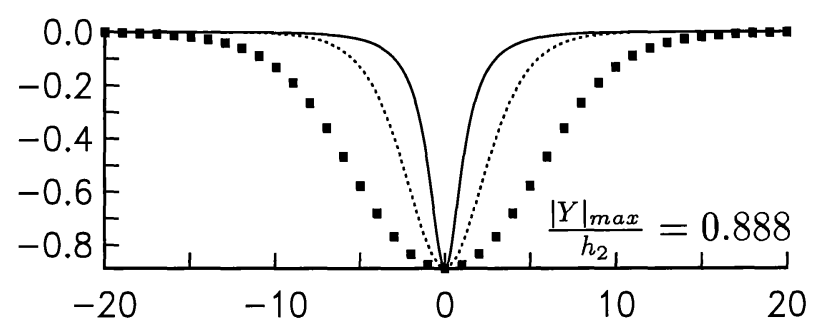

c)

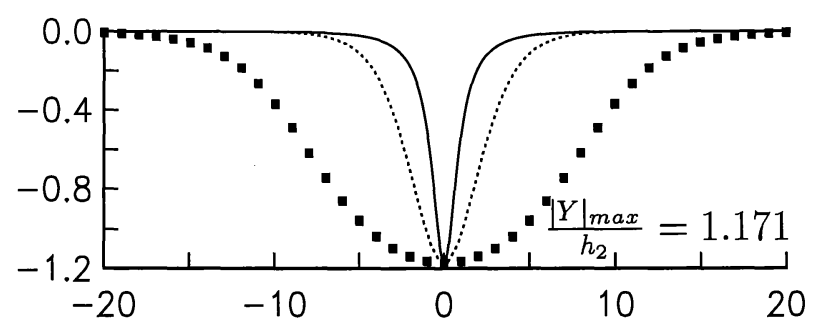

d)

$x / h_{2}$

Figure 4: Profiles of solitary waves obtained by fully nonlinear (black squares), KdV (dotted line) and finite-depth (solid line) theories. $h_{1} / h_{2}=4$ in all cases. a) $|Y|_{\max } / h_{2}=$ $0.15, \mu=0.9$. b) $|Y|_{\max } / h_{2}=0.7, \mu=0.9$. c) $|Y|_{\max } / h_{2}=0.888, \mu=0.8114$. d) $|Y|_{\max } / h_{2}=1.171, \mu=0.8114$. (Note the differences in vertical scale.) 


\section{Transcritical flow over topography}

We then apply the model to transcritical two-layer flow over a bottom topography. There are several questions concerning this subject: Under which conditions is the flow unsteady? Another aspect is upstream influence in stratified flows, which in part can be addressed by the present two-layer model. Furthermore, for which conditions may transcritical flow over topography generate upstream solitary waves? These topics have been discussed in earlier works describing observations in nature, laboratory experiments and theoretical models. The theoretical models used are basically hydraulic nonlinear theory, see e.g. Baines (1984), Grimshaw and Smyth (1986), or weakly nonlinear dispersive models, see e.g. Miles $(1979,1981)$, Melville and Helfrich (1987), Zhu, Wu and Yates (1987). These methods have limited validity with regard to nonlinearity and dispersion, however, and give unrealistic predictions for finite amplitude and moderate wave length.

Melville and Helfrich (1987), below referred to as MH, described two sets of experiments on interfacial transcritical flow at a geometry. They compared the experiment with simulations by the forced $\mathrm{KdV}$ equation and an extended $\mathrm{KdV}$-model, where in the latter a cubic nonlinearity was taken into account in addition to the usual quadratic nonlinearity. They got, however, a poor agreement between theory and experiments when the ratio between the depths of the layers was one to four, but better agreement when the ratio was one to two. We shall apply our method to the set of experiments of $\mathrm{MH}$ where the disagreements between the $\mathrm{KdV}$-models and the observations were most severe, and we shall find a very good agreement between the fully nonlinear model and the experiments. Furthermore, we shall apply our model to investigate generation of upstream solitary waves in transcritical flows.

The data of the set of experiments of $\mathrm{MH}$ we shall compare with are as follows: $\rho_{2}=$ $0.8 \mathrm{~g} / \mathrm{cm}^{3}, h_{2}=3 \mathrm{~cm}, \rho_{1}=0.986 \mathrm{~g} / \mathrm{cm}^{3}, h_{1}=12 \mathrm{~cm}$. The effect of a bottom topography was simulated in the experiments by moving a geometry in the upper layer with a profile given by $y=h_{2}-H_{0} \operatorname{sech}^{2}(K x)$, where $K H_{0}=0.1989$ (this geometry is the $2 \mathrm{D}$ counter-part of Nansen's ship). The height of the geometry was determined by $H_{0} / h_{0}=0.5$, with the reference depth $h_{0}=h_{1} h_{2} /\left(h_{1}+h_{2}\right)$. (This means that $H_{0} / h_{2}=0.4$.) $\mathrm{MH}$ applied a tank of length $\mathrm{L}=15 \mathrm{~m}\left(\mathrm{~L} / h_{2}=500\right)$ and recorded the displacement of the interface after $5 \mathrm{~m}$ $\left(x / h_{2}=166\right)$, hereafter referred to as station 1 , and the horizontal velocity in the lower layer after $9.25 \mathrm{~m}\left(x / h_{2}=308\right)$, hereafter referred to as station 2 . At the latter station also photographs of the interface were taken. (We note that $\mathrm{MH}, \mathrm{p} .36$ give $H_{0}=5.1 \mathrm{~cm}$, which, however, does not seem to fit with the other data given).

We use all the above conditions in our simulations, except at the upper boundary, where the free surface is replaced by a rigid wall.

\subsection{Speed and elevation of the upstream disturbance}

As reference speed we shall use the linear shallow water speed given by

$$
c_{0}=\left[\frac{\left(\rho_{1}-\rho_{2}\right) g h_{1} h_{2}}{\rho_{1} h_{2}+\rho_{2} h_{1}}\right]^{1 / 2}
$$

In $\mathrm{MH}$ (eq. 2.6) their reference speed is given by $\tilde{c}_{0}=\left[g h_{0}\left(\rho_{1}-\rho_{2}\right) / \rho_{0}\right]^{1 / 2}$, where $\rho_{0}$, however, is not explitly defined. It seems likely that $\rho_{0}$ shall be chosen equal to $\rho_{1}$, and we have used this reference density in the comparisons. This gives a very good agreement 
between their experiments and our comparisons, see figure 5. If $\rho_{0}$ is chosen such that $\tilde{c}_{0}=c_{0}$ we still get a fair agreement.

In the first comparison we consider the speed $c$ of the leading disturbance of the interface as function of the speed $U$ of the geometry. This is one of the most robust quantities to measure and compare, and was in the experiments determined by recording the elapsed time of the leading disturbance between the recording stations. We have followed the same procedure in extracting $c$ from the computations, and the excellent agreement is shown in figure $5 \mathrm{a}$.

Next we show in figure $5 \mathrm{~b}$ the maximum elevation $|Y|_{\text {max }}$ of the upstream disturbance. We use the same vertical scale as $\mathrm{MH}$ (with $h_{0}$ as vertical reference length). The computations show that $|Y|_{\max }$ is somewhat growing as the geometry is moving downward the wavetank, and we therefore present results when the waves have come to the far end of the tank. The agreement between the model and the experiments is in general very good, but we note that the model somewhat underpredicts the measured elevations when the speed of the geometry exceeds $c_{0}$. For Froude number $U / c_{0}=1.2$ we compute a significantly smaller value for $|Y|_{\text {max }}$ than measured. We are not sure about the reason for this disagreement, but we note that this Froude number is close to the upper limit of the transcritical domain in this example, where the maximal elevation as function of the Froude number drops significantly.

$\mathrm{MH}$ noted that the curves for $\left(c-c_{0}\right) / \dot{c}_{0}$ and $|Y|_{\max } / h_{0}$ in figure 5 with the scaling shown should coincide, according to KdV-theory. These curves have, however, quite different forms. Our computations confirm the experimental observations.

\subsection{Profiles of the interface}

We then consider simulations of the interface elevation and compare with reproduced recordings from $\mathrm{MH}$, figure 10 . In the experiments are recorded the elevation $Y$ at station 1 and the horizontal velocity $u_{1}$ in the lower layer at station 2 . All recordings are functions of time. The computed interface profiles $Y(x)$ are shown for time instants when the geometry is (approximately) at station 1 (solid line) and at station 2 (dashed line). The geometry started at $x=0$. (We use the same vertical scale as MH.)

In figures 6-8 are shown comparisons for three different choices of $U$, viz. $U / c_{0}=0.81$ $\left(U / \tilde{c}_{0}=0.88\right), U / c_{0}=0.94\left(U / \tilde{c}_{0}=1.02\right), U / c_{0}=1.14\left(U / \tilde{c}_{0}=1.24\right)$. In the cases when $U / c_{0}<1$, figures $6-7$, both theory and experiments show that an upstream undular depression is generated, with a number of local crests which is increasing with time. The elevation behind the geometry reaches a maximum level at about $40 \%$ of the thickness of the upper layer, and is continously growing horisontally with time. For $U / c_{0}=1.14$ (figure 8) theory and experiment show that a depression of appreciable magnitude is generated close to the geometry, with speed slightly larger than $U$. The flow is clearly unsteady, as an elevation downstream and a corresponding volume upstream is continously developing. The upstream depression becomes a solitary wave after sufficiently large time.

All computations reproduce the experiments with striking agreement. The number of local wave crests of the upstream disturbance which is passing the recording stations, and the elevation behind the geometry are almost exactly reproduced by the theory. We have run convergence checks confirming the computations shown. We have no explanation why the theoretical depression systematically is somewhat smaller than the recordings, but we speculate that the deviation between the computations and the measurements fall within the error bounds of the interface recorder used in the experiments. 

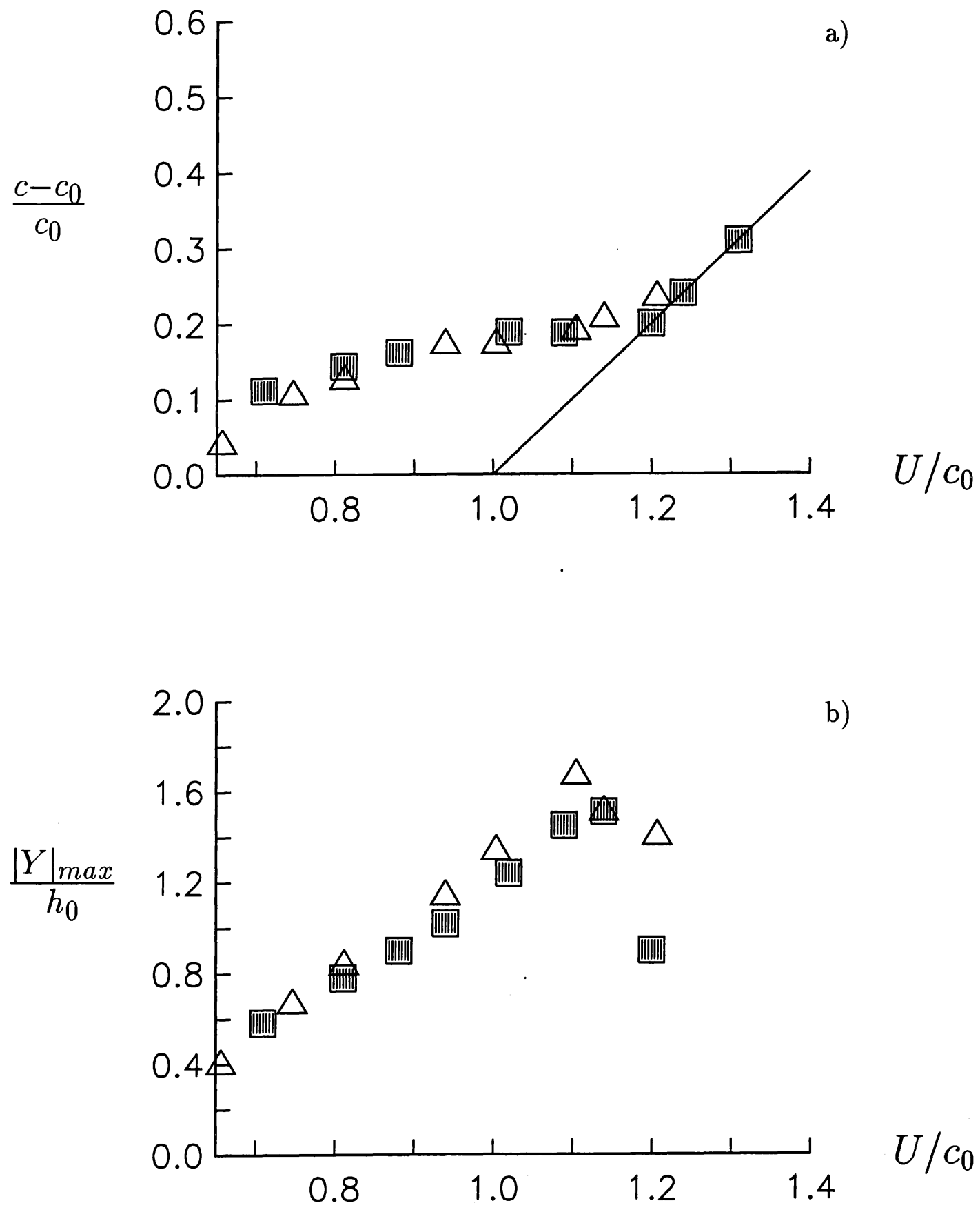

Figure 5: a) Excess speed $\left(c-c_{0}\right) / c_{0}$ and b) maximum elevation of leading upstream disturbance vs. $U / c_{0}$. Black squares: Present theory. Triangles: Experiments by Melville and Helfrich, figure 11. Solid line in a) marks solution moving with the same speed as the geometry. 


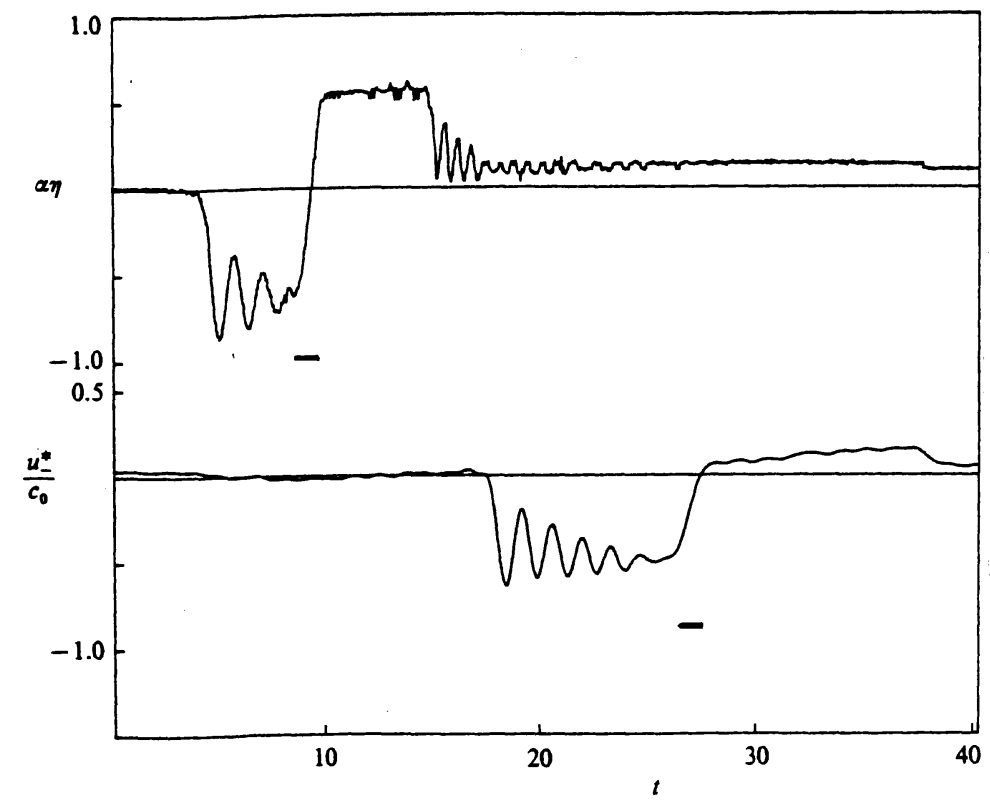

a)

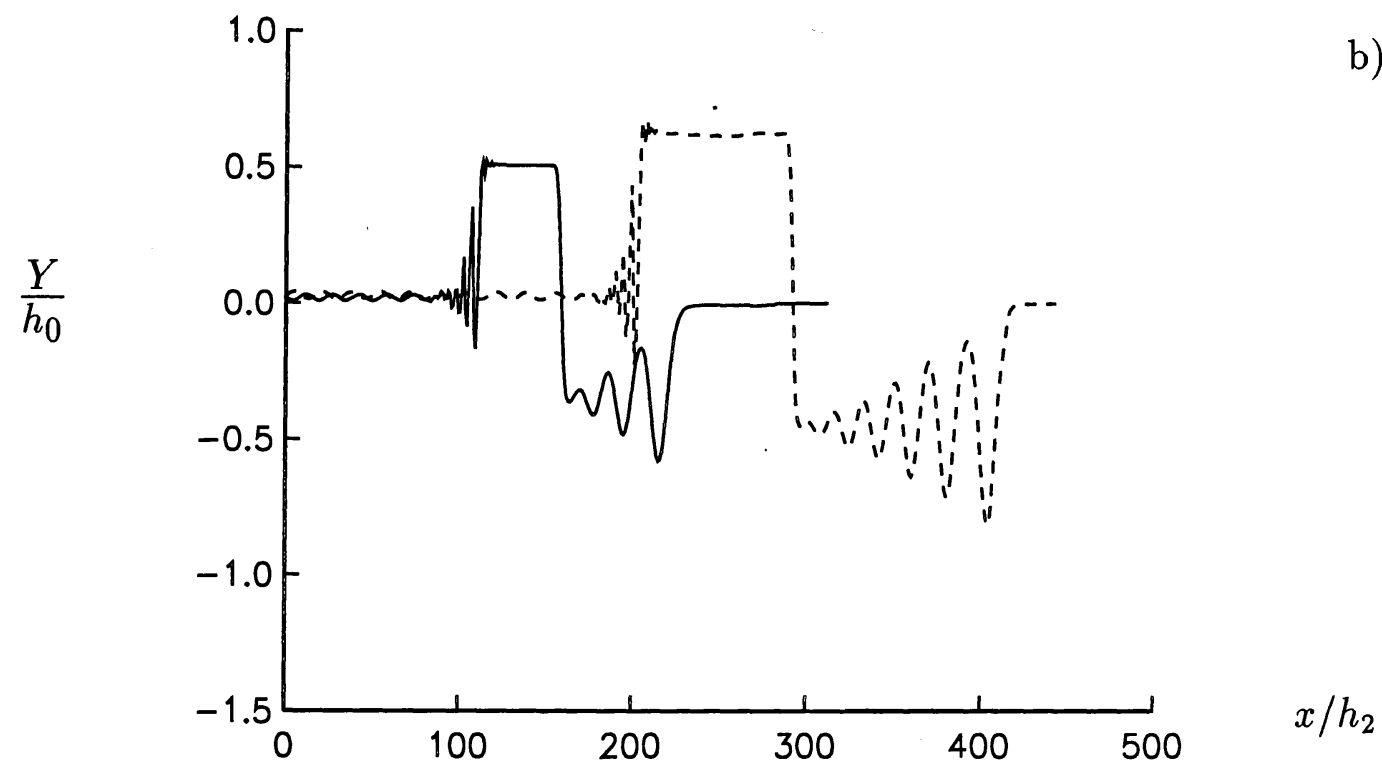

Figure 6: Elevation of interface. $U / c_{0}=0.81$. a) Experimental recordings reproduced from $\mathrm{MH}$ figure $10 \mathrm{a}\left(U / \tilde{c}_{0}=0.881\right)$. b) Present method. Same vertical scale as in the experiments $\left(h_{0}=0.8 h_{2}\right) \cdot t\left(g / h_{2}\right)^{1 / 2}=480$ (solid line), $t\left(g / h_{2}\right)^{1 / 2}=870$ (dashed line). $\mu=0.8114, h_{1} / h_{2}=4, \Delta t\left(g / h_{2}\right)^{1 / 2}=0.5, \Delta T\left(g / h_{2}\right)^{1 / 2}=5$ (smooting interval), $\Delta x / h_{2}=1, N=501, N_{B}=80, \alpha=1$. 


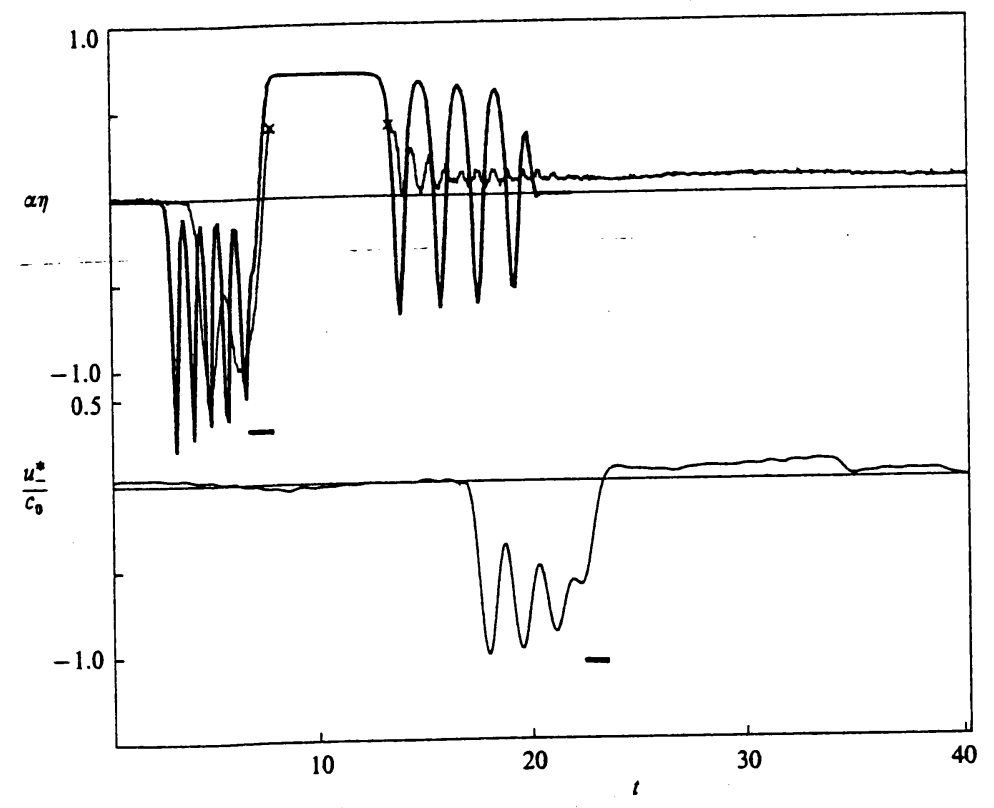

a)

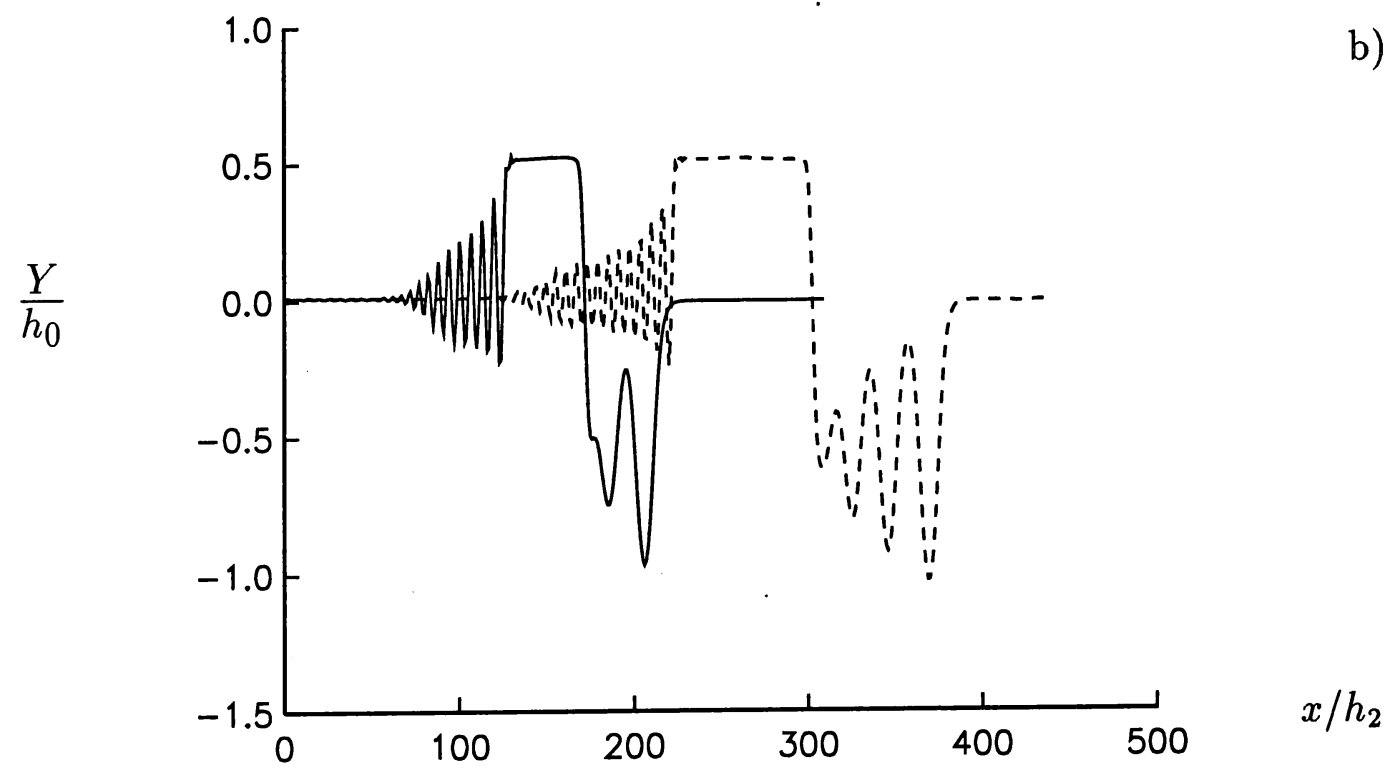

Figure 7: Same as figure 6 but $U / c_{0}=0.94$. a) $\mathrm{MH}$ figure $10 \mathrm{~b}\left(U / \tilde{c}_{0}=1.02\right)$. Fat solid line: Solution by the forced KdV-equation. b) Present method. $t\left(g / h_{2}\right)^{1 / 2}=450$ (solid line), $t\left(g / h_{2}\right)^{1 / 2}=780$ (dashed line). 


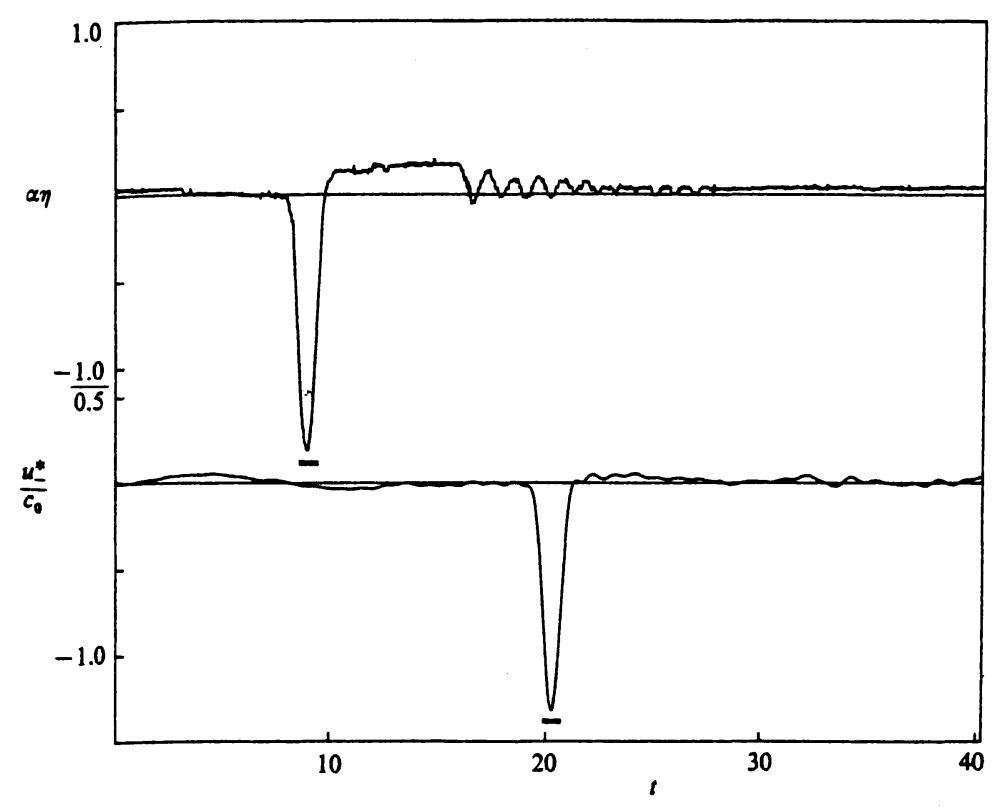

a)

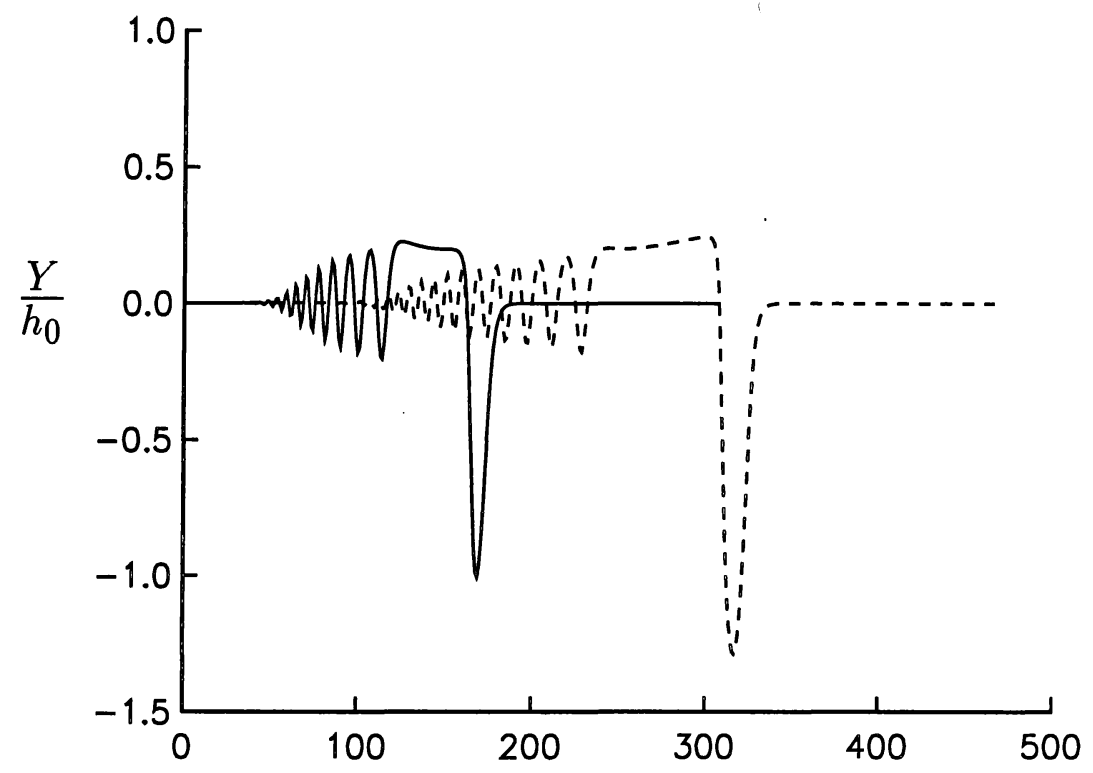

b)

$$
x / h_{2}
$$

Figure 8: Same as figure 6 but $U / c_{0}=1.14$. a) $\mathrm{MH}$ figure $10 \mathrm{c}\left(U / \tilde{c}_{0}=1.24\right)$. b) Present method. $t\left(g / h_{2}\right)^{1 / 2}=360$ (solid line), $t\left(g / h_{2}\right)^{1 / 2}=660$ (dashed line). 
In figure $7 \mathrm{a}$ is also shown simulations by $\mathrm{MH}$ using the $\mathrm{KdV}$ equation, which exhibits twice as many peaks of the upstream undular depression than the observations in the wave tank and the fully nonlinear computations.

\subsection{Regime of unsteady transcritical flow}

We find that the flow is unsteady for $0.38 \sim<U / c_{0}<\sim 1.2$. In this regime the simulations show that the fluid volume upstream, being an undular depression or a train of solitary waves (see §5.4), and the length of the elevation behind the geometry are steadily increasing with time. When $U / c_{0}>1.2$, supercritical flow is obtained. The transient waves due to the upstart of the geometry are then swept downstream and steady state is reached. When $U / c_{0}<0.4$, a train of steady lee waves are generated behind the geometry. Analytical estimates of the unsteady regime of transcritical flow are derived by means of asymptotic theories, for free surface flows by Miles (1986), and for interfacial waves by $\mathrm{MH}$. The latter estimate predicts in the present example a lower limit which is quite unrealistic and a range of transcritical flow being too wide.

\subsection{Upstream solitary waves}

When the speed of the geometry is in the transcritical regime but less than $c_{0}$, an undular upstream depression is generated. This is also true when $U$ slightly exceeds $c_{0}$. In the remaining part of the transcritical regime we find that solitary waves propagating upstream are generated by the geometry. In order to investigate the latter regime more closely we fix the speed to $U / c_{0}=1.09$ and vary the height of the geometry from a small to a large value. More presicely, we let the geometry be determined by $y=h_{2}-\tilde{H}_{0} \operatorname{sech}^{2}(K x)$, where $\tilde{H}_{0}$ takes the values $\tilde{H}_{0}=0.125 H_{0}, 0.25 H_{0}, 0.5 H_{0}, H_{0} . \quad\left(H_{0}=0.4 h_{2}\right.$ as before.) Increasing the value of $\tilde{H}_{0}$ gives a larger forcing of the flow, but it also corresponds to imposing stronger nonlinearity to the problem. We show in figure 9 the resulting profiles of the interface after nondimensional times $t\left(g / h_{2}\right)^{1 / 2}=1020,1500$, when the geometry is at $x / h_{2}=469,689$, respectively. The maximal depressions in the figure correspond to those of the solitary waves shown in figure 4 .

In the first case the flow is very close to being supercritical, and the depression at the position of the geometry is rather small. When we increase $\tilde{H}_{0}$ from $0.125 H_{0}$ to $0.25 H_{0}$ the change is rather large, however. Now the flow has become unsteady, an elevation behind the geometry is continously developing, and the depression slightly ahead of the body attains a rather large peak which slowly develops into a solitary wave.

The next two examples show the initial phases of generation of upstream solitary waves. We observe in the last case (figure $9 \mathrm{~d}$ ) that one solitary wave is already generated before the geometry has passed the location at $x / h_{2}=500$, corresponding to the length of the wave tank used in the experiments by $\mathrm{MH}$, which means that solitary waves should, according to the simulations, have been observed in their laboratory experiments for this depth ratio if they had taken relevant measurements at the end of the wave tank. In order to more closely examine the properties of the simulated upstream waves we compare with steady solitary wave solutions obtained by solving the equations, without presence of the geometry, in a frame of reference following one wave, given the value of $|Y|_{\max } / h_{2}$. The comparisons are displayed in figures $10 \mathrm{a}-\mathrm{b}$ and show that the time simulations have produced leading waves having exactly the same forms as the steadily progressing solutions. In the first case $\left(\tilde{H}_{0}=0.5 H_{0}\right)$ the amplitude is $|Y|_{\text {max }} / h_{2}=0.888$, 


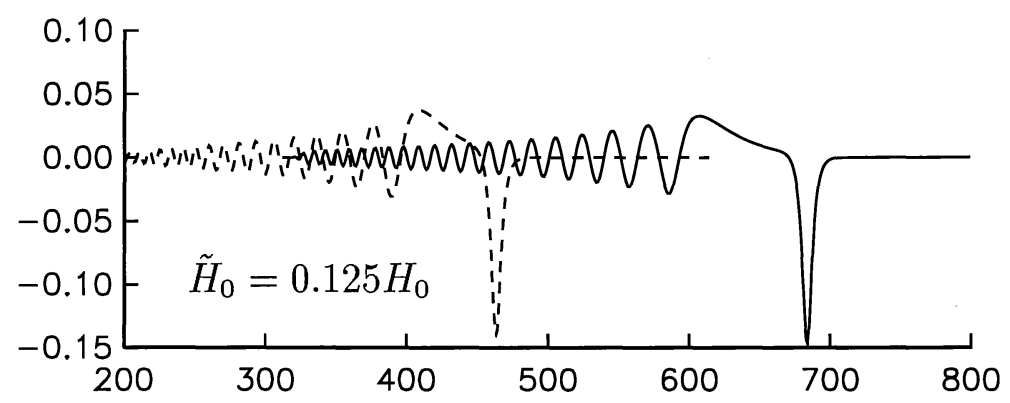

a)

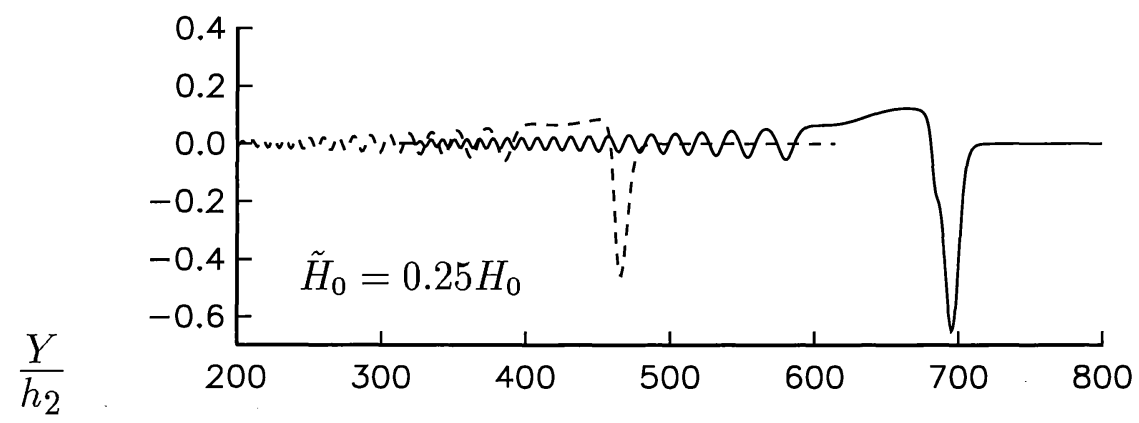

b)

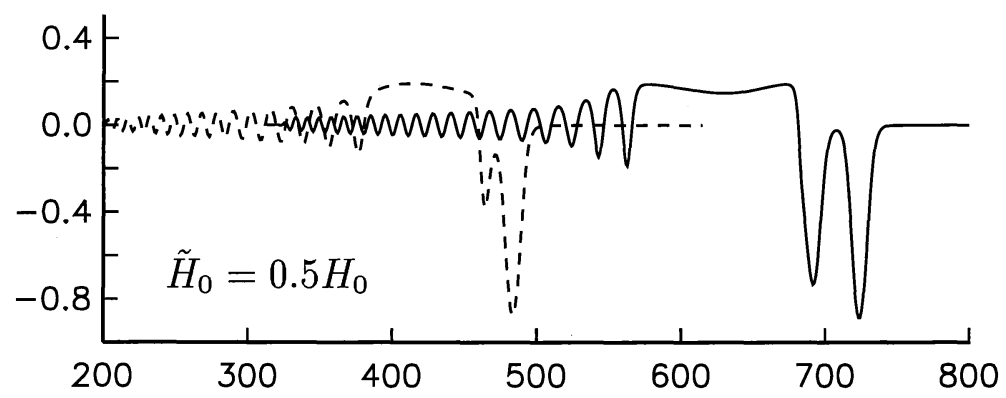

c)

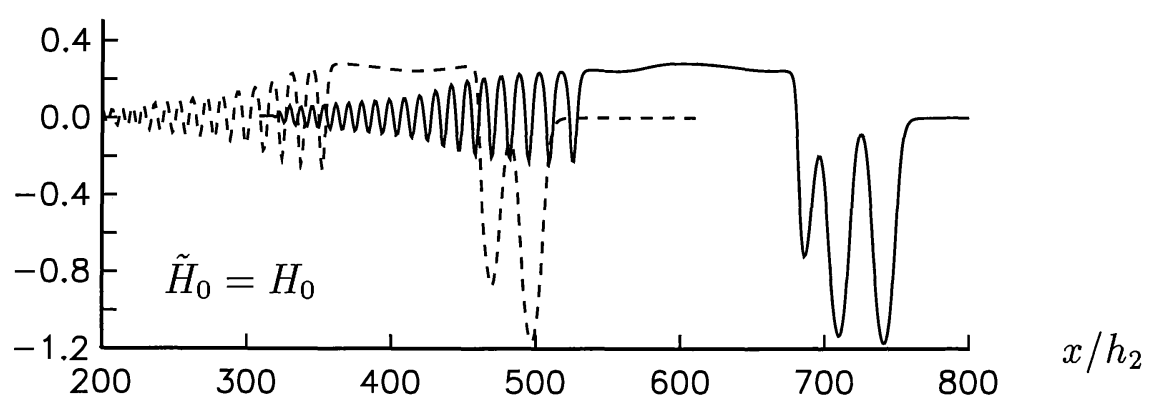

d)

Figure 9: Elevation of interface. $F r=1.09$. Increasing height $\tilde{H}_{0}$ of moving geometry, i.e. $\tilde{H}_{0} \operatorname{sech}^{2} K x$. $t\left(g / h_{2}\right)^{1 / 2}=1020$ (dashed line) $t\left(g / h_{2}\right)^{1 / 2}=1500$ (solid line). a) $\tilde{H}_{0}=0.125 H_{0}$. b) $\tilde{H}_{0}=0.25 H_{0}$. c) $\tilde{H}_{0}=0.5 H_{0}$. d) $\tilde{H}_{0}=H_{0} . \mu=0.8114, h_{1} / h_{2}=4$, $\Delta t\left(g / h_{2}\right)^{1 / 2}=0.5, \Delta T\left(g / h_{2}\right)^{1 / 2}=5$ (smooting interval), $\Delta x / h_{2}=1, N=501, N_{B}=80$, $\alpha=1$. (Note the differences in vertical scale.) 

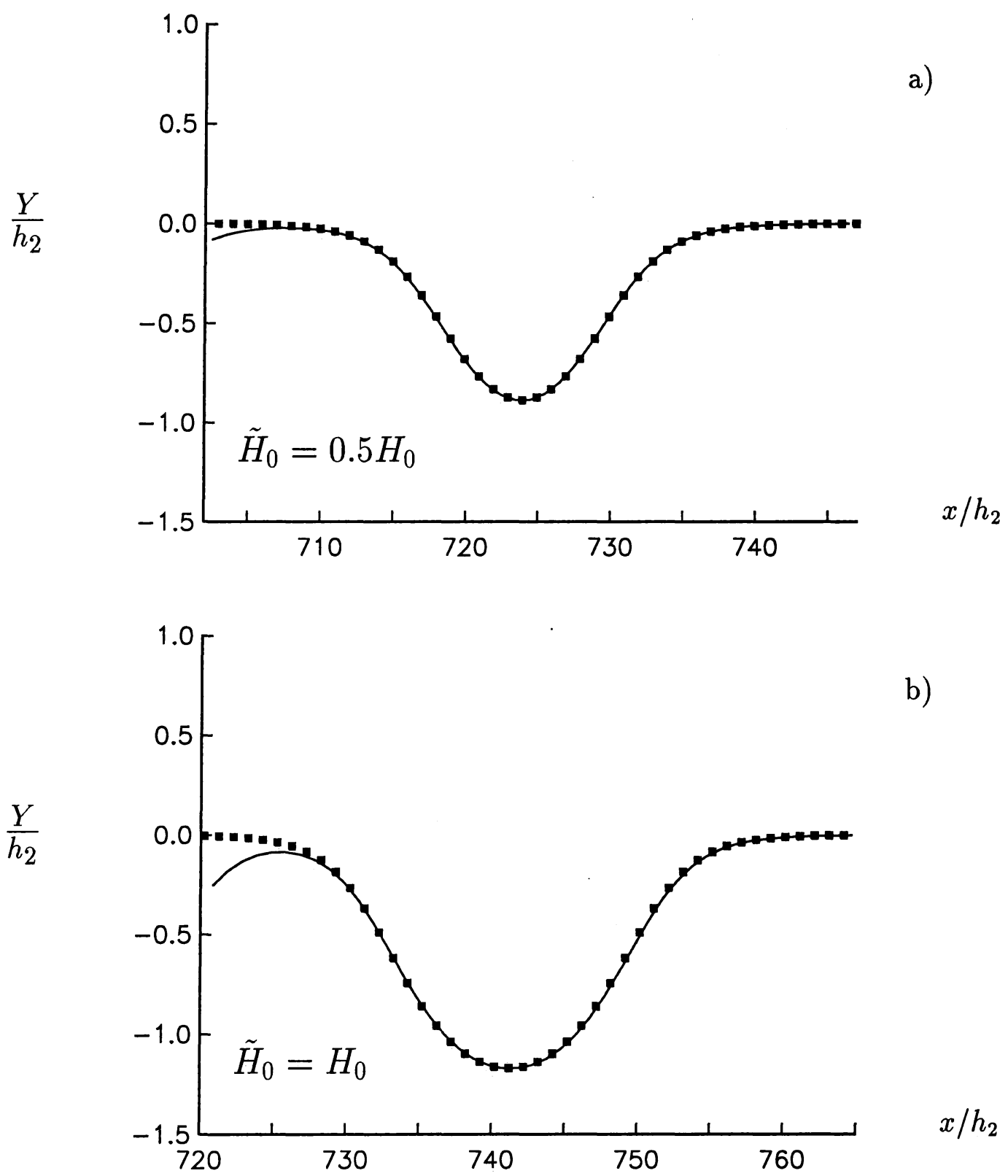

Figure 10: Comparison between simulated leading upstream wave (solid line) and solitary wave solution, steady profile (black squares). a) Same as figure 9c (geometry given by $\left.0.5 H_{0} \operatorname{sech}^{2} K x\right) .|Y|_{\max } / h_{2}=0.888$. b) Same as figure $9 \mathrm{~d}$ (geometry given by $H_{0} \operatorname{sech}^{2} K x$ ). $|Y|_{\max } / h_{2}=1.171$. 
and we find that the propagation velocity in the time simulation is $c_{s i m} /\left(g h_{2}\right)^{1 / 2}=0.5007$, while the solution of the steady equations gives $c_{\text {steady }} /\left(g h_{2}\right)^{1 / 2}=0.5008$. In the other case $\left(\tilde{H}_{0}=H_{0}\right)$ the corresponding results are $|Y|_{\text {max }} / h_{2}=1.171, c_{s i m} /\left(g h_{2}\right)^{1 / 2}=0.5091$ and $c_{\text {steady }} /\left(g h_{2}\right)^{1 / 2}=0.5092$. This very good agreement documents that the leading upstream waves indeed may be termed solitary waves.

Finally we consider a different example where $h_{2} / h_{1}=4$ (a thick layer above a thin), $\mu=0.7873$ and a half elliptical bottom topography with horizontal half-axis $10 h_{1}$ and vertical half-axis $0.1 h_{1}$ is moving with speed $U / c_{0}=1.1$ in the lower layer. This topography has about the same volume as the previous, but imposes weaker nonlinearity on the problem, since the height of the topography now is only $10 \%$ of the thinner layer. We have performed a very long time simulation with this configuration (5520 time steps), and show the results in figure 11. The generation rate of solitary waves is higher than in the former example, but the amplitude is somewhat less. At nondimensional time $t\left(g / h_{1}\right)^{1 / 2}=1080$ two solitary waves are generated, at $t\left(g / h_{1}\right)^{1 / 2}=1920$ almost four, and at $t\left(g / h_{1}\right)^{1 / 2}=2760$ almost six waves. The depression behind the topography stabilizes at a level of $80 \%$ of the initial thickness of the lower fluid. The solitary waves all have the same amplitude, within a variation of $0.3 \%$. Upon comparing with the solution of a steady profile with $|Y|_{\text {max }} / h_{2}=0.869$, we find a striking agreement between the computed profiles, see figure 11d, and the wave speed; $c_{\text {sim }} /\left(g h_{1}\right)^{1 / 2}=0.5192, c_{\text {steady }} /\left(g h_{1}\right)^{1 / 2}=0.5191$. Thus, the simulated waves may be regarded as a train of solitary waves. The comparison document the high accuracy of the time simulations.

We find that the distance between the peaks are almost the same, except for a small oscillation of the order less than $1 \%$ of a mean value of about $28.5 h_{1}$, which indicates that a weak interaction is taking place between the individual waves. For $t\left(g / h_{1}\right)^{1 / 2}=2760$, the distance between the leading and the second wave is somewhat larger.

\section{Conclusion}

We have described a fully nonlinear time stepping method for unsteady motion of a twolayer fluid. Essential parts of the method are the use of Taylor series expansions of the prognostic equations, application of spacial finite difference formulae of high order and application of Cauchy's theorem to solve the Laplace equation. The latter is found to be superior compared to other methods with regard to avoid instability, a result which is partly explained by a stability analysis showing that the scheme is neutrally stable for linear flows. Details of the numerical implementation is described and convergence of the method is documented in several examples. The spacial step length, $\Delta x$, must be less than twice the smallest thickness of thinner layer, in order to maintain analyticity of the function representing the image of the interface, see eq. (20). $\Delta x$ is in most of the computations set equal to the thickness of the thinner layer. We may use a relatively large time step but still achieve a high accuracy of the computations. The method is computationally very efficient and is suitable for long time simulations. The velocity profiles in the fluids are inherent in the formulation and may easily be extracted from the computations (we have not shown velocity profiles here).

Fully nonlinear solitary wave profiles are compared with available experiments, finding very good agreement. Upon comparing with weakly nonlinear theories ( $\mathrm{KdV}, \mathrm{BO}$ and finite-depth) we find that these theories have quite limited ranges of validity, and that they predict unrealistic wave shapes when the maximum elevation becomes comparable 


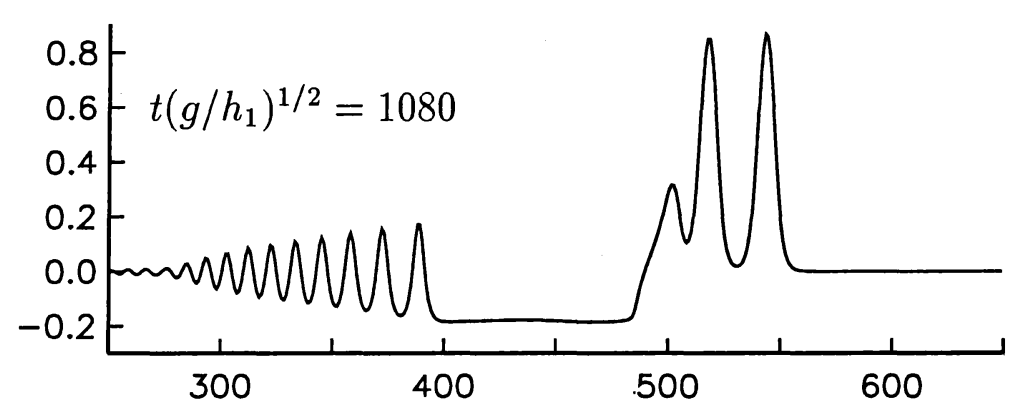

a)

b)

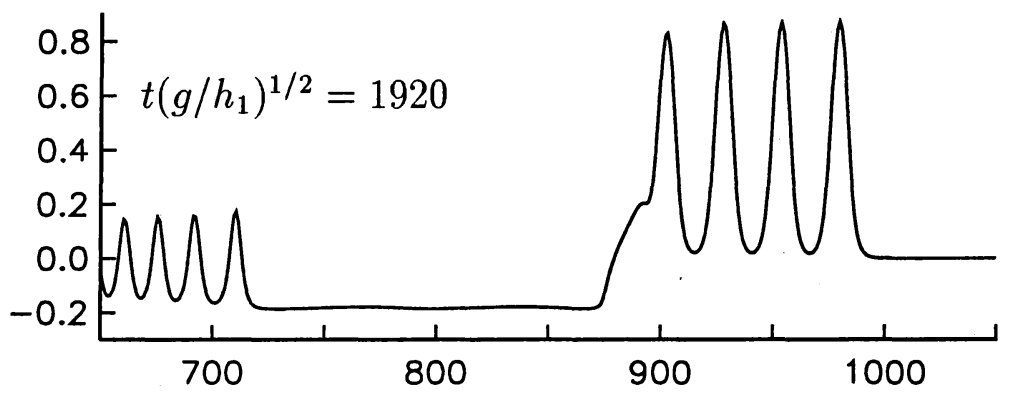

c)
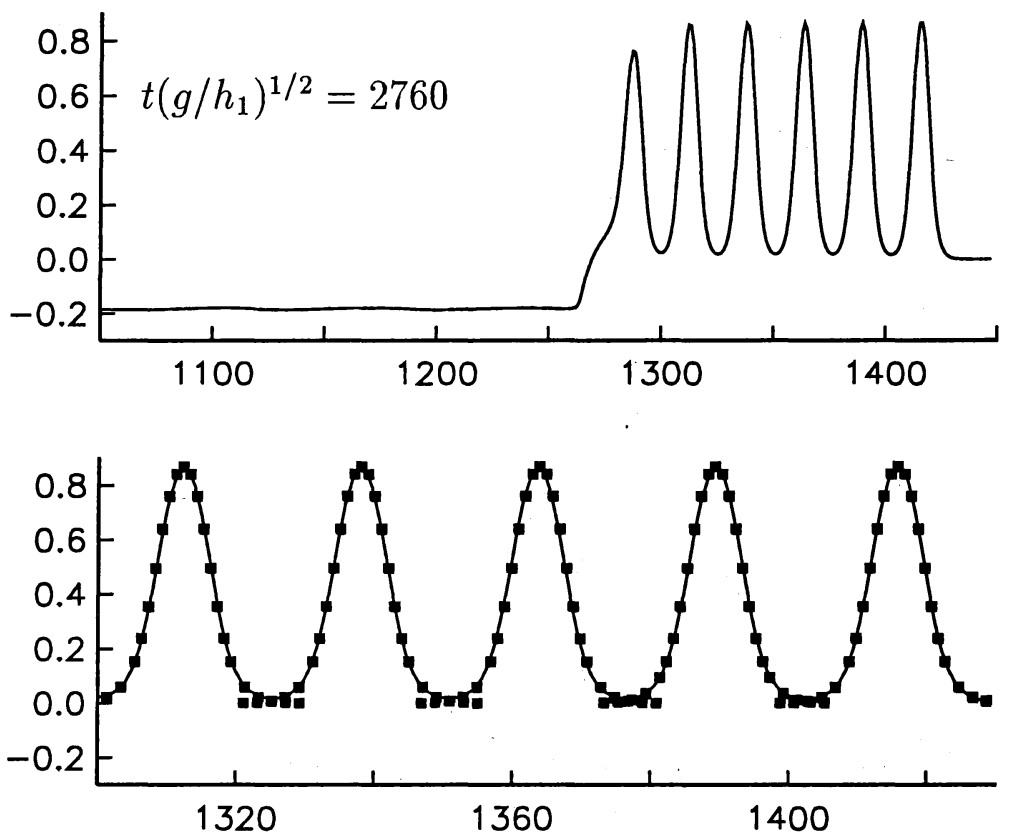

d)

$x / h_{1}$

Figure 11: Generation of upstream solitary waves. Moving bottom topography being a half ellipse, horizontal half-axis $10 h_{1}$, vertical half-axis $0.1 h_{1} . U / c_{0}=1.1, \mu=0.7873$, $h_{2} / h_{1}=4$. a) $t\left(g / h_{1}\right)^{1 / 2}=1080$. b) $t\left(g / h_{1}\right)^{1 / 2}=1920$. c) $t\left(g / h_{1}\right)^{1 / 2}=2760$. d) Close up of figure c), black squares mark steady solitary wave solution with $|Y|_{\text {max }} / h_{1}=0.869$. $\Delta t\left(g / h_{1}\right)^{1 / 2}=0.5, \Delta T\left(g / h_{1}\right)^{1 / 2}=3$ (smooting interval), $\Delta x / h_{1}=1, N=501, N_{B}=80$, $\alpha=0$. 
to the thickness of the thinner layer.

We apply the model to simulate a set of experiments by Melville and Helfrich (1987) on unsteady transcritical flow at a moving geometry, were $\mathrm{KdV}$ theory was reported to give unrealistic results. We find here a satisfactory agreement between the fully nonlinear theory and the experiments. Furthermore, the unsteady transcritical regime is identified, where an undular depression is generated when the speed of the geometry is less than a value which somewhat exceeds the critical speed, and a train of solitary waves is generated otherwise. A corresponding elevation of constant level develops behind the geometry. Then generation of upstream solitary waves by a moving geometry is in some examples investigated in detail, finding that trains of solitary waves with appreciable heights are propagating upstream. We have compared the simulated waves with solitary wave profiles obtained by solving the nonlinear equations in a frame of reference following the waves, where the propagation speed is determined from the equations. There is a very good agreement between the simulated and steady profiles. Even the propagation speed is reproduced in the time simulations with a relative accuracy of $0.02 \%$.

In the examples discussed in $\S 5$ the ratio between the depths of the layers is one to four. In all examples we find that a moving geometry generates upstream disturbances with rather large depression (elevation). We have performed simulations for other geometries with smaller heights, imposing a corresponding weaker nonlinearity to the problem. Still we experience that waves with rather large amplitudes are generated (the volume of the geometry cannot be too small). Upon comparing with the results in $\S 4$ we find that the flow is clearly outside the ranges of validity of the $\mathrm{KdV}$ and finite-depth theories. We find that the same conclusion applies to the BO-theory (results not shown here). Our results thus indicate that weakly nonlinear theories have quite limited applications in modelling unsteady transcritical two-layer flows when $h_{1} / h_{2}>>1$ (or $h_{2} / h_{1}>>1$ ), and that a fully nonlinear method in general is required for this purpose. There may be exceptions for very long geometries with very small height.

The method may be used to investigate collision properties of steep interfacial solitary waves, and their interaction with a fixed geometry in the ocean or a bottom topography (work is in progress). Furthermore, we may use the method to simulate tidal generated interfacial waves at a sill, and the transient development of a large hump into solitary waves.

At the free surface we have used the rigid lid approximation. The method may, however, be generalized to also model the motion of the free surface.

This research was supported by The Research Council of Norway through Reserch Fellowships for H.A.F and P.O.R. and a grant of computing time (Programme for Supercomputing). 


\section{A Pseudo Lagrangian derivatives at the interface}

To derive integral equations for $\partial \phi_{1 \nu} / \partial t, \partial \Upsilon_{\xi} / \partial t, \partial^{2} \phi_{1 \nu} / \partial t^{2}$ and $\partial^{2} \Upsilon_{\xi} / \partial t^{2}$, pseudo Lagrangian derivatives of the kinematic boundary condition at the interface are required. Taking the pseudo Lagrangian derivative of (2) we find

$$
\frac{D_{\times}}{d t}\left(\mathbf{v}_{1} \cdot \hat{\mathbf{n}}-\mathbf{v}_{2} \cdot \hat{\mathbf{n}}\right)=\hat{\mathbf{n}} \cdot \frac{D_{\times}}{d t}\left(\mathbf{v}_{1}-\mathbf{v}_{2}\right)+\left(\mathbf{v}_{1}-\mathbf{v}_{2}\right) \cdot \frac{D_{\times} \hat{\mathbf{n}}}{d t}=0
$$

where a scaled normal vector $\hat{\mathbf{n}}=\left|z_{\xi}\right| \mathbf{n}=\left(-Y_{\xi}, X_{\xi}\right)$ at $I$ is introduced for convenience. Noting that

$$
\frac{D_{\times} \hat{\mathbf{n}}}{d t}=\frac{\partial}{\partial t}\left(-Y_{\xi}(\xi, t), X_{\xi}(\xi, t)\right)=\frac{\partial}{\partial \xi}\left(-Y_{t}(\xi, t), X_{t}(\xi, t)\right)=\left(-v_{\times \xi}, u_{\times \xi}\right)
$$

eq. (45) gives

$$
\begin{aligned}
& \frac{\partial \phi_{1 \nu}}{\partial t}-\frac{\partial \phi_{2 \nu}}{\partial t}=y_{\xi}\left[u_{\times}\left(u_{1 x}-u_{2 x}\right)+v_{\times}\left(v_{1 x}-v_{2 x}\right)\right] \\
& -x_{\xi}\left[u_{\times}\left(v_{1 x}-v_{2 x}\right)+v_{\times}\left(-u_{1 x}+u_{2 x}\right)\right]+\left(u_{1}-u_{2}\right) v_{\times \xi}+\left(v_{1}-v_{2}\right) u_{\times \xi}
\end{aligned}
$$

which is used to derive integral equations for $\partial \phi_{1 \nu} / \partial t, \partial \Upsilon_{\xi} / \partial t$, similar to (28)-(29).

Next we consider

$$
\frac{D_{\times}^{2}}{d t^{2}}\left(\mathbf{v}_{1} \cdot \mathbf{n}-\mathbf{v}_{2} \cdot \mathbf{n}\right)=0
$$

By carrying out the differentiation we obtain

$$
\begin{aligned}
& \frac{\partial^{2} \phi_{1 \nu}}{\partial t^{2}}-\frac{\partial^{2} \phi_{2 \nu}}{\partial t^{2}}=-\hat{\mathbf{n}} \cdot\left\{\mathbf{v}_{\times} \cdot \nabla\left(\frac{\partial \mathbf{v}_{1}}{\partial t}-\frac{\partial \mathbf{v}_{2}}{\partial t}\right)+\frac{\partial}{\partial t}\left[\mathbf{v}_{\times} \cdot \nabla\left(\mathbf{v}_{1}-\mathbf{v}_{2}\right)\right]\right. \\
& \left.-\mathbf{v}_{\times} \cdot \nabla\left[\mathbf{v}_{\times} \cdot \nabla\left(\mathbf{v}_{1}-\mathbf{v}_{2}\right)\right]\right\}+2 \frac{D_{\times} \hat{\mathbf{n}}}{d t} \cdot \frac{D_{\times}}{d t}\left(\mathbf{v}_{1}-\mathbf{v}_{2}\right)-\left(\mathbf{v}_{1}-\mathbf{v}_{2}\right) \cdot \frac{D_{\times}^{2} \hat{\mathbf{n}}}{d t^{2}}
\end{aligned}
$$

where $D_{\times} \hat{\mathbf{n}} / d t$ is determined by (46) and

$$
\frac{D_{\times}^{2} \hat{\mathbf{n}}}{d t^{2}}=\left(-\left(\frac{D_{\times} v_{\times}}{d t}\right)_{\xi},\left(\frac{D_{\times} u_{\times}}{d t}\right)_{\xi}\right)
$$

Eq. (49) is used to derive integral equations for $\partial^{2} \phi_{1 \nu} / \partial t^{2}, \partial^{2} \Upsilon_{\xi} / \partial t^{2}$, similar to (28)-(29).

Pseudo derivatives of $\psi$ are found by differentiating the dynamical boundary condition at the interface.

\section{B The integral equations on discrete form}

The discrete forms of the integral equations (28), (29) and (30) are given by

$$
\begin{aligned}
\pi \Upsilon_{\xi}\left(\xi^{\prime}\right) & =\frac{2}{\mu+1} \sum_{\xi=1}^{N} \mathcal{B}\left(\xi^{\prime}, \xi\right) \psi_{\xi}(\xi)+\frac{\mu-1}{\mu+1} \sum_{\xi=1}^{N} \mathcal{B}\left(\xi^{\prime}, \xi\right) \Upsilon_{\xi}(\xi) \\
& +\sum_{\xi=1}^{N}\left[\mathcal{A}_{1}\left(\xi^{\prime}, \xi\right)-\mathcal{A}_{2}\left(\xi^{\prime}, \xi\right)\right] \phi_{1 \nu}(\xi) \\
& +\frac{1}{1+\mu} \sum_{\xi=1}^{N}\left[-\mathcal{B}_{1}\left(\xi^{\prime}, \xi\right)-\mathcal{B}_{2}\left(\xi^{\prime}, \xi\right)\right] \psi_{\xi}(\xi)
\end{aligned}
$$




$$
\begin{aligned}
& +\frac{1}{1+\mu} \sum_{\xi=1}^{N}\left[-\mu \mathcal{B}_{1}\left(\xi^{\prime}, \xi\right)+\mathcal{B}_{2}\left(\xi^{\prime}, \xi\right)\right] \Upsilon_{\xi}(\xi) \\
& +\sum_{\xi=1}^{N_{B}}\left[-\mathcal{A}\left(\xi^{\prime}, \xi\right)+\mathcal{A}_{1}\left(\xi^{\prime}, \xi\right)\right] \phi_{1 \nu}(\xi) \\
& +\sum_{\xi=1}^{N_{B}}\left[\mathcal{B}\left(\xi^{\prime}, \xi\right)-\mathcal{B}_{1}\left(\xi^{\prime}, \xi\right)\right] \phi_{1 \xi}(\xi) \quad(z \text { on } I) \\
& \pi(1+\mu) \phi_{1 \nu}\left(\xi^{\prime}\right)=\sum_{\xi=1}^{N} \mathcal{A}\left(\xi^{\prime}, \xi\right) \psi_{\xi}(\xi)-\psi_{\xi \xi}\left(\xi^{\prime}\right)+(1-\mu) \sum_{\xi=1}^{N} \mathcal{B}\left(\xi^{\prime}, \xi\right) \phi_{1 \nu}(\xi) \\
& +\frac{1}{1+\mu} \sum_{\xi=1}^{N}\left[\mathcal{A}_{1}\left(\xi^{\prime}, \xi\right)+\mu \mathcal{A}_{2}\left(\xi^{\prime}, \xi\right)\right] \psi_{\xi}(\xi) \\
& +\frac{\mu}{1+\mu} \sum_{\xi=1}^{N}\left[\mathcal{A}_{1}\left(\xi^{\prime}, \xi\right)-\mathcal{A}_{2}\left(\xi^{\prime}, \xi\right)\right] \Upsilon_{\xi}(\xi) \\
& -\sum_{\xi=1}^{N}\left[-\mathcal{B}_{1}\left(\xi^{\prime}, \xi\right)+\mu \mathcal{B}_{2}\left(\xi^{\prime}, \xi\right)\right] \phi_{1 \nu}(\xi) \\
& +\sum_{\xi=1}^{N_{B}}\left[\mathcal{A}\left(\xi^{\prime}, \xi\right)+\mathcal{A}_{1}\left(\xi^{\prime}, \xi\right)\right] \phi_{1 \xi}(\xi) \\
& +\sum_{\xi=1}^{N_{B}}\left[\mathcal{B}\left(\xi^{\prime}, \xi\right)+\mathcal{B}_{1}\left(\xi^{\prime}, \xi\right)\right] \phi_{1 \nu}(\xi) \quad(z \text { on } I) \\
& \pi \phi_{1 \xi}\left(\xi^{\prime}\right)=\sum_{\xi=1}^{N}\left[-\mathcal{A}\left(\xi^{\prime}, \xi\right)+\mathcal{A}_{1}\left(\xi^{\prime}, \xi\right)\right] \phi_{1 \nu}(\xi) \\
& +\frac{1}{1+\mu} \sum_{\xi=1}^{N}\left[\mathcal{B}\left(\xi^{\prime}, \xi\right)-\mathcal{B}_{1}\left(\xi^{\prime}, \xi\right)\right] \psi_{\xi}(\xi) \\
& +\frac{\mu}{1+\mu} \sum_{\xi=1}^{N}\left[\mathcal{B}\left(\xi^{\prime}, \xi\right)-\mathcal{B}_{1}\left(\xi^{\prime}, \xi\right)\right] \Upsilon_{\xi}(\xi) \\
& +\sum_{\xi=1}^{N_{B}}\left[-\mathcal{A}\left(\xi^{\prime}, \xi\right)+\mathcal{A}_{1}\left(\xi^{\prime}, \xi\right)\right] \phi_{1 \nu}(\xi)+\phi_{1 \nu \xi}\left(\xi^{\prime}\right) \\
& +\sum_{\xi=1}^{N_{B}}\left[\mathcal{B}\left(\xi^{\prime}, \xi\right)-\mathcal{B}_{1}\left(\xi^{\prime}, \xi\right)\right] \phi_{1 \xi}(\xi) \quad(z \text { on } B)
\end{aligned}
$$

where $\mathcal{A}+i \mathcal{B}$ is given by $(35)$ and $\mathcal{A}_{j}+i \mathcal{B}_{j}(j=1,2)$ by

$$
\mathcal{A}_{j}+i \mathcal{B}_{j}=\left(\frac{z_{\xi}^{\prime}}{z^{*}-z^{\prime}+(-1)^{j} 2 i h_{j}}\right)^{*}, \quad j=1,2
$$




\section{References}

[1] Amick, C. J. And Turner, R. E. L. A global theory of internal solitary waves in two-fluid systems. Trans. Am. Math. Soc., 298, 1986.

[2] Apel, J. R., Holbrook, J. R., Liu, A. K. And Tsai, J. The Sulu Sea internal soliton experiment. J. Phys. Oceanography. 15, 1985.

[3] Baines, P. A unified description of two-layer flow over topography. J. Fluid Mech., 146, 1984.

[4] Baker, G.R., Meiron, D.I. and Orszag, S.A. Generalized vortex methods for free-surface flow problems. J. Fluid Mech., 123, 1982.

[5] Benjamin, T.B. Internal waves of permanent form in fluids of great depth. J. Fluid Mech., 29, 1967.

[6] Davis, R. E. AND Acrivos, A. Solitary internal waves in deep water. J. Fluid Mech., 29, 1967.

[7] DoLD, J.W. An efficient surface-integral algorithm applied to unsteady gravity waves J. Comp. Phys., 103, 1992.

[8] Dold, J. W. And Peregrine, D. H. An efficient boundary-integral equation method for steep unsteady water waves. Numerical Methods for Fluid Dynamics, 2, ed. K. W. Morton and M. J. Baines, Clarenden Press, Oxford, 1985.

[9] Eliassen, A. Vilhelm Bjerknes and his students. Ann. Rev. Fluid Mech., 14, 1982.

[10] Eliassen, A. And Fjørtoft, R. The interface method for calculating the temporal evolution of a two-fluid system. Frontiers of Science, Annals of the New York Academy of Sciences, 661, 1992.

[11] Farmer, D. M. And Smith, J. D. Tidal interaction of stratified flow with a sill in Knight Inlet. Deep-Sea Res., 27A, 1980.

[12] Grimshaw, R. H. J. And Smyth, N. Resonant flow of a stratified fluid over topography. J. Fluid Mech., 169, 1986.

[13] Holyer, J.Y. Large amplitude progressive interfacial waves. J. Fluid Mech., 93, 1979.

[14] Joseph, R.I. Solitary waves in a finite depth fluid. J. Phys. A: Math. Gen., 10, 1977.

[15] Keulegan, G. H. Characteristics of internal solitary waves. J. Res. Natl. Bureau of Standards, 51, 1953.

[16] Koop, C. G. And Butler, G. An investigation on internal solitary waves in a two-fluid system. J. Fluid Mech., 112, 1981.

[17] Kubota, T. And Ko, D.R.S. And DobBs, L.D. Weakly-nonlinear, long internal gravity waves in stratified fluids of finite depth. J. Hydronautics, 12, 1978. 
[18] Long, R. R. Solitary waves in one and two fluid systems. Tellus, 8, 1956.

[19] Meiron, D. I. And Saffman, P. G. Overhanging interfacial garvity waves of large amplitude. J. Fluid Mech., 120, 1983.

[20] Melville, W. K. And Helfrich, K. R. Transcritical two-layer flow over topography. J. Fluid Mech., 178, 1987.

[21] Miles, J. W. On internal solitary waves. Tellus, 31, 1979.

[22] Miles, J. W. On internal solitary waves II. Tellus, 33, 1981.

[23] Ono, H. Algebraic Solitary Waves in Stratified Fluids. J. Phys. Soc. Japan, 39, 1975 .

[24] Pullin, D.I. AND Grimshaw, R.H.J. Finite-amplitude solitary waves at the interface between two homogeneous fluids. Phys. Fluids, 31, 1988.

[25] Roberts, A. J. A stable and accurate numerical method to calculate the motion of a sharp interface between fluids. IMA J. Appl. Math., 31, 1983.

[26] Segur, H. and Hammack, J. L. Soliton models of long internal waves. J. Fluid Mech., 118, 1982.

[27] Staquet, C. AND Sommeria, J. Internal waves, turbulence and mixing in stratified flows: a report on Euromech Colloquium 339. J. Fluid Mech., 314, 1996.

[28] Shelley, M.J. A study of singularity formation in vortex-sheet motion by a spectrally accurate vortex method. J. Fluid Mech., 244, 1992.

[29] VAnden-Broeck, J.-M. Numerical calculation of gravity-capillary interfacioal waves of finite amplitude. Phys. Fluids, 23, 1980.

[30] Vinje, T. And Brevig, P. Numerical simulation of breaking waves. Adv. Water Res., 4, 1981.

[31] WU, T. Y. Generation of upstream advancing solitons by moving disturbances. $J$. Fluid Mech., 184, 1987.

[32] Zhu, J., Wu, T. Y. AND YATES, G. T. Internal solitary waves generated by moving disturbances. 3rd Int. Symp. on Stratified Flows, California Inst. of Tech., Pasadena, California, 3-5 February, 1987. 